\title{
On the instability and
} constraints of the interaction between number representation and spatial attention in healthy humans: A concise review of the literature and new experimental evidence

\author{
E. Fattorini ${ }^{*, \dagger, 1}$, M. Pinto ${ }^{*, \dagger, 1}$, S. Merola*, ${ }^{*}$, M. D’Onofrio ${ }^{*}, \dagger$, F. Doricchi ${ }^{*, \dagger, 2}$ \\ *Università degli Studi di Roma 'Sapienza', Rome, Italy \\ ${ }^{\dagger}$ Fondazione Santa Lucia IRCCS, Rome, Italy \\ ${ }^{2}$ Corresponding author: Tel.: +39-6-49917929, e-mail address: fabrizio.doricchi@uniromal.it
}

\section{Abstract}

The relationship between number and space representation is still one of the most debated topics in studies of mathematical cognition. Here we offer a concise review of two important behavioral effects that have pointed out the use of a spatially left-to-right oriented mental number line (MNL) in healthy participants: the SNARC effect and the attentional SNARC effect (Att-SNARC). Following a brief summary of seminal investigations on the introspective properties of the MNL, we review recent empirical evidence and theories on the functional origin of the SNARC effect, where upon left/right response choices faster reaction times are found for small numbers with left-side responses and for large numbers with right-side responses. Then we offer a summary of the studies that have investigated whether the mere perception of visual Arabic numbers presented at central fixation can engender spatially congruent lateral shifts of attention, ie, leftward for small numbers and rightward for large ones, ie, the Att-SNARC effect. Finally, we summarize four experiments that tested whether the Att-SNARC depends on an active rather than passive processing of centrally presented digit cues. In line with other recent studies, these experiment do not replicate the original Att-SNARC and show that the

\footnotetext{
${ }^{1}$ These authors contributed equally to the study.
} 
mere perception of Arabic numerals does not trigger automatic shifts of attention. These shifts are instead found when the task requires the explicit left/right spatial coding of digit cues, ie, Spatial Att-SNARC (Fattorini et al., 2015b). Nonetheless, the reliability of the Spatial Att-SNARC effect seems not as strong as that of conventional SNARC effects where left/right codes are mapped onto responses rather than directly mapped on digit cues. Comparing the magnitude of digits to a numerical reference, ie, "5," also produced a Magnitude Comparison Att-SNARC that was weaker than the spatial one. However, the reliability of this Magnitude Comparison Att-SNARC should be considered with caution because, like in a study by Zanolie and Pecher (2014), we recently failed to replicate this effect in a separate behavioral-eventrelated potentials study in preparation (Fattorini et al., 2015a). All together the results from the present series of experiments support the hypothesis that spatial coding is not an intrinsic part of number representation and that number-space interaction is determined by the use of stimulus- or response-related spatial codes in the task at hand.

\section{Keywords}

Numbers, Space, SNARC, Attentional SNARC

\section{INTRODUCTION}

\subsection{INTROSPECTIVE NUMBER FORMS: THE MENTAL NUMBER LINE}

One of the most intriguing issues in mathematical cognition is the functional and anatomical relationship between the processing of number magnitudes and the processing of spatial information. This problem was originally highlighted by the seminal introspective reports collected by Francis Galton at the turn of the 19th century and summarized in two Nature articles (Galton, 1880a,b). In the first article, Galton described the different imaginative number-forms that in some healthy humans, herein after defined "number synestheses," were automatically elicited upon hearing or perceiving number-words. Number synestheses had the striking introspective experience of a visual mental number line (MNL). In some of these subjects, the MNL was oriented in the horizontal direction, while others reported vertically oriented MNL or MNL with alternating horizontal, vertical, diagonal, or curved segments. Galton also sharply noted that despite these varying forms, all synetheses reported the presence of landmarks, turns, breaks, "woolly lumps," or changes in luminosity and color at the points separating tens and hundreds on the MNL. In his second and unfortunately less quoted paper (Galton, 1880b), Galton added the subtle and important observation that the frame of spatial reference of the MNL varied among synestheses: in some cases the MNL moved in synchrony with eye and head movements, suggesting its reliance on retinotopic and/or head centered coordinates, while in others it maintained an invariant position in mental space independently of the direction of eye and head movements. The pioneering observations reported by Galton were confirmed and expanded by his contemporary Jacques Bertillon. 
Bertillon published his observations in three different papers published in " $L a$ Nature" (Bertillon, 1880, 1881, 1882). In his third report, Bertillon first noted the tendency of some healthy humans to spontaneously organize the series of the months of the year and days of the week in visual number forms (see Eagleman, 2009, for an empirical update of this specific issue). Two more recent studies have adopted the administration of detailed questionnaires to reinvestigate the presence of the MNL and its association with mental lines for other ordinal sequences (eg, days in a week, months in a year, and letters in the alphabet) in large samples of unselected healthy adults (Seron et al., 1992) or in selected samples of number/letter-color and number/letter-taste synesthetes and nonsynesthetes (Sagiv et al., 2006). The main conclusions of the study by Seron et al. (1992) were that the form of the MNL is stable within participants and that number forms like the MNL are not specifically limited to number sequences and might have instead developed for elements that, as in the case of numbers "constitute well-delineated subparts of the lexicon, are sequentially organized and have been learned by rote in a conventional order during childhood." Seron et al. (1992) showed that number forms like the MNL are more prevalent in number/letter-color synestheses and that, confirming the suggestion by Seron et al. (1992), the MNL tends to occur with visuospatial forms for other ordinal sequences (eg, days, months, and letters) "which suggests that it is the ordinal nature of numbers rather than numerical quantity that gives rise to this particular mode of representation."

\subsection{THE SNARC EFFECT}

Inquiries in introspective reports of "number forms" in synestheses deserve important credits for demonstrating the reliability of the "phenomenological" interaction between number and space and for generating the entire new field of research in the neural bases of synesthesia (for review, see Price and Mattingley, 2013). However, these studies left untested the possibility that the use of a spatial representation of number magnitudes can also be elicited in healthy and not necessarily synestheses participants. The true turning point that moved the study of the links between number and space beyond the introspective level and that pointed out the use of a left-to-right spatially organized MNL in healthy participants was the discovery of the SNARC effect by Dehaene et al. (1993; for preliminary observations, see also Dehaene et al., 1990). These authors observed that in tasks requiring left vs right motor responses, judgements of number parity (eg, Parity Judgement, PJ: odd or even?), or number magnitude (eg, Magnitude Comparison, MC: smaller or larger than 5?; Dehaene et al., 1990) are faster when left-side responses are associated with small magnitudes and right-side responses with large magnitudes rather than vice versa. In the last 20 years a large number of investigations (see Wood et al., 2008) have confirmed the strength and reliability of the SNARC effect. These findings have led to the widespread belief that in the human brain number magnitudes have an inherent spatial organization that follows reading habits, so that, for example, in 
western culture small numbers are automatically coded as being placed to the left of large ones or, as in the original words by Dehaene et al. (1993), that the spatial representation of numbers "bears a natural and seemingly irrepressible correspondence with the natural left-right coordinates of external space." Nonetheless, it is important to note that such a diffused belief stands in striking contrast with the persisting lack of an agreement on the functional origin of the SNARC effect itself and the level of cognitive processing at which a spatially organized MNL is generated during the performance of SNARC tasks. Both the PJ- and the MC-version of the SNARC task require the processing of relevant number-related features, the association of these features to motor responses and the selection of motor responses on the basis of contrasting left vs right spatial codes that define the position of the response in space. Accordingly, different functional interpretations of the SNARC effect have situated the genesis of the left-to-right organized MNL at different points along the continuum that ranges from the perception of numbers to the performance of the motor response associated with task-relevant number features (Cohen Kadosh et al., 2008; Wood et al., 2008). A first interpretation (Hubbard et al., 2005) embraces the idea that, though subject to cultural and task-context factors, the SNARC effect occurs because spatial codes are a constituent part of number representation. Accordingly, numbers occupy "default" spatial positions on the mental equivalent of a left-to-right organized ruler and, therefore, "numbers automatically elicit task-, modality-, and effector-independent spatial representations, even when these spatial representations are not strictly relevant to the task."

A second type of interpretation emphasizes that the SNARC effect is determined by culturally acquired associations between "left/right" and "small/large" codes (Gevers et al., 2010; Proctor and Cho, 2006; Santens and Gevers, 2008). More specifically, Proctor and Cho (2006) have argued that a culturally based "positive polarity" is usually assigned to concepts like "right" and "large," while a negative polarity is assigned to concepts like "left" and "small." Classifying a number as "larger" than 5 is therefore easier and faster when the spatial response code is "right," ie, when the response code has the same polarity of the "large" stimulus code. In contrast the same decision is slower when the response code is "left," which has the opposite polarity of "large." It is worth noting that this explanation proposes that the SNARC effect does not depend on an internal and visual spatial left-to-right oriented MNL but, rather, on the "verbal coding of space" (Gevers et al., 2010).

A third type of interpretation emphasizes the role played by response selection processes based on spatial left/right codes (Ishihara et al., 2006; Muller and Schwarz, 2007). This "response-related" interpretation of the SNARC effect is supported by converging evidence from different event-related potentials (ERPs) studies. These show that the SNARC effect arises at the response-related stage during the selection of the left vs right response key, rather than at an early stage of perceptual or visual imagery processing (Gevers et al., 2006; Keus and Schwarz, 2005). More specifically, a difference between potentials evoked in the number/response compatible (small number/left response and large number/right response) as compared to the incompatible number/response condition (small number/right response and large 
number/left response) was only found at the level of the stimulus-locked lateralized response potentials (LRP). The latency of compatible LRP was significantly shorter than that of incompatible LRP (Gevers et al., 2006; Keus and Schwarz, 2005) and the amplitude of the compatible LRP higher than that of the incompatible LRP (Keus and Schwarz, 2005). The LRP is recorded over the motor cortex and marks the controlled selection of a motor response. In the case of the SNARC effect, it reflects the mapping of the mental representation of the number onto the response. It is worth noting that though placing the functional origin of the SNARC effect at a late processing stage the response-related account assumes an inherent "default" link between small numbers and the spatial code "left" and between large numbers and the spatial code "right." As an example in the dual-route network model of the SNARC effect proposed by Gevers et al. (2006), within the sector of the network simulating the "fast unconditional route" that incorporates long-term links between magnitude and spatial representation, the node "smaller" projects, by default, to the response node "left" and the node "larger" projects to response node "right." Later in this paper, we shall put forward the different hypothesis (see also Fattorini et al., 2015b) that these links are not automatically or constantly active by "default" and that they are rather engendered by the use of the spatial labels "left" and "right" in the selection of the motor response or in the explicit spatial classification of number magnitudes presented during numerical tasks.

Finally, a further interpretation proposed by van Dijck and Fias (2011) postulates that the SNARC effect is driven by a temporary and flexible association between number and space at the working memory stage rather than by a stable spatial organization of number magnitudes in long-term semantic memory. The working memory account comes from the evidence that the SNARC effect is abolished when spatial (Herrera et al., 2008) or phonological information (van Dijck et al., 2009) has to be maintained in working memory. These results suggest that the working memory load interferes with the spatial representation of numbers. van Dijck and Fias (2011) expanded these findings by asking participants to perform a Parity Judgement SNARC task on a previously memorized sequence of five random numbers. The authors found that, rather than being merely determined by the position occupied along the left-to-right MNL representing the sequence of ascending integers, the SNARC effect was based on the relative position occupied by numbers in the memorized sequence, so that responses with left hand were faster for numerical items at the beginning of the sequence, while responses with right hand were faster for those at the end of the sequence. Accordingly, the authors concluded that "systematic ordering of maintained information rather than numerical magnitude is the crucial determinant of the SNARC effect."

\subsection{THE ATTENTIONAL SNARC EFFECT}

Fischer et al. (2003) highlighted a behavioral effect that pointed at an inherent and response-independent link between number magnitude and the orienting of spatial attention. These authors ran two experiments, one in a sample of 15 healthy 
participants (Experiment 1) and one in a sample of 10 participants (Experiment 2). Both experiments required unimanual responses to visual dot-targets presented in the left or in the right side of space. Targets were preceded by small-magnitude (1 or 2) or large-magnitude digit cues ( 8 or 9 ) presented at central fixation at varying cuetarget intervals (CTIs). In both experiments faster RTs to left-side targets were found when these were preceded by small magnitude cues and faster RTs to right-side targets were found when these were preceded by large magnitude cues. This attentional advantage, defined Attentional SNARC effect (Att-SNARC), was significant at 500 and $750 \mathrm{~ms}-\mathrm{CTI}$ and was found despite participants were informed that digit cues were irrelevant to target detection and did not predict target location. These findings seemingly suggested that the mere perception of Arabic numbers engenders shifts of spatial attention that are directionally congruent with number magnitude, ie, with the position occupied by numbers on the MNL: therefore, small number magnitudes should produce automatic leftward shifts of attention, whereas large numbers rightward shifts.

The observation of the Att-SNARC had a powerful influence in reinforcing the assumption of a tight link between number and space representation. Nonetheless, the results of the many ensuing studies dedicated to the assessment of the AttSNARC have provided conflicting results and, at the same time, important qualifications of the specific task conditions that might determine the Att-SNARC. Among the first replication studies, those run by Ristic et al. (2006) and Galfano et al. (2006) strongly emphasized that, whenever found, the Att-SNARC is far from being an automatic effect and it is rather driven by strategic top-down factors. This conclusion was based on the finding that the direction of the AttSNARC can be reversed just by asking participants to imagine a MNL running in the right-to-left rather than left-to-right direction (Ristic et al., 2006). In the same vein Galfano et al. (2006) showed that the putative automatic shifts of attention highlighted by the Att-SNARC were labile and could be easily counteracted, because the Att-SNARC was reversed when participants were asked to shift attention leftward in response to large numbers and rightward in response to small numbers. In addition, in a study run by one of the authors of the original study by Fischer et al. (2003), the Att-SNARC was only found at one $(500 \mathrm{~ms})$ out of the two CTIs $(500 \mathrm{~ms}$ and $750 \mathrm{~ms})$ at which the effect was originally observed (Dodd et al., 2008). Studying a sample of 26 healthy participant and using 50, 200, 400, and $650 \mathrm{~ms}$-CTIs, also Bonato et al. (2009) failed to replicate the Att-SNARC. Although these negative finding could be ascribed to the use of CTIs that are generally shorter than those at which the Att-SNARC was originally described, it is important to note that at the longer CTI (ie, $650 \mathrm{~ms}$ ) Bonato et al. (2009) found identical RTs for spatially congruent and incongruent targets $(315 \mathrm{~ms}$, see table 1 in Bonato et al., 2009). The use of relatively short CTIs might have also caused both the negative results reported by Jarick et al. (2009), who examined a sample of 12 participants using six different CTIs that ranged from 50 to $500 \mathrm{~ms}$, and those reported by Hubbard et al. (2009, Experiment 2) who also tested 12 participants with a set of short CTIs (range 50-500 ms). Unfortunately in both reports it is 
not made clear whether the Att-SNARC was eventually present at the longest CTIs. Mixed results were reported by Ranzini et al. (2009) in a combined RTs-ERP study. During the task, trials with central arrow cues pointing left or right were alternated with central numerical cues $(1,2,8$, and 9). Lateral targets were presented at three different CTIs: 300, 400, or $500 \mathrm{~ms}$. At the behavioral level, the authors observed no significant Att-SNARC $(p=0.065)$, whereas at the electrophysiological levels cue- and target-related activity evoked in numerical trials seemed to reveal number-space congruency effects. Nonetheless, in the cue period (EDAN and ADAN components) these effects were much smaller and short lasting than those elicited during trials with spatial arrow cues. In the target period congruency effects were observed at occipital sites with arrow cues and at central-parietal sites with number-cues. Though intriguing these ERPs results are difficult to interpret due to the lack of a significant Att-SNARC effect. In addition, these results cannot be directly compared to those originally obtained by Fischer et al. (2003), because number and directional arrow cues pointing left/right were continuously alternated in consecutive trials, so that the spatial codes conveyed by arrow cues could have contaminated the processing of numerical cues. In another ERP study, Salillas et al. (2008), presented lateral targets at a fixed $450 \mathrm{~ms}$-CTI from central numerical cues, and compared ERPs between targets appearing at positions congruent or incongruent with the magnitude of the central numerical cue. At variance with the original Att-SNARC task, no speeded detection of lateral targets was required in order to avoid contamination of target-related ERP activity on the part of processes related to motor response. The authors reported significant number-space congruency effects on the P1 and P3 waves. Unfortunately, also in this case the interpretation of ERP results is in part difficult because no matching Att-SNARC could be documented by the study. More recently, Schuller et al. (2015) investigated cue- and targetrelated ERPs during the detection of lateral red/green targets that were preceded by central digit cues, ie, 1, 2, 8, and 9. Participants were required to choose between a left or a right key press as a function of target color. An Att-SNARC effect was observed only for targets in the left side of space, while the general Att-SNARC did not reach significance because a nonsignificant reversed Att-SNARC was found for targets in the right side of space. ERPs effect were similar to those documented by Ranzini et al. (2009): nonetheless, as cautiously noted by the authors, the use of left/right spatial codes in the selection of the responses to targets introduced spatialcompatibility effects that spuriously added to the putative attentional effects triggered by central digit cues. In conclusion, current ERPs evidences are promising though further replications with the original Att-SNARC task are needed to reach unequivocal conclusions on the link between behavioral and electrophysiological effects. We note that a similar problem seems present with fMRI inquiries on the Att-SNARC. As an example, Goffaux et al. (2012) found no Att-SNARC and no lateralized activations in parietal areas that are typically involved in leftward (ie, right hemisphere) and rightward (ie, left hemisphere) shifts of spatial attention but, at the same time, observed lateralized activations in occipital areas in response to small and large magnitude digit cues. 
Among the most recent psychophysical studies, the investigations by van Dijck et al. (2014) and Zanolie and Pecher (2014) are particularly relevant to the assessment of the reliability of the Att-SNARC, because both of these studies faithfully replicated the paradigm used by Fischer et al. (2003, Experiment 2). van Dijck et al. (2014) adopted a conservative methodological approach and estimated "a priori" the number of participants, ie, 31, that were necessary to reach a power of 0.90 based on the effect sizes observed by Fischer et al. (2003). Forty-three participants were then examined (Experiment 1) and no Att-SNARC was found. In a different experiment from the same study (Experiment 2) a significant Att-SNARC was instead found when digit cues belonged to a previously memorized short sequence of four numerical items. In this case, items at the beginning of the sequence elicited leftward shifts of attention and those at the end of the sequence rightward shifts. The direction of shifts was entirely uncoupled from number magnitude and only dependent on the position of the digit cue in the sequence. The authors concluded that these evidences suggest a close link between the serial and ordered arrangement of the information maintained in working memory and spatial attention (see also, van Dijck and Fias, 2011; van Dijck et al., 2013). Zanolie and Pecher (2014) performed two series of three experiments. In both series (Experiments 1 and 4) no Att-SNARC was observed with the original paradigm (Fischer et al., 2003). In two other experiments ( 2 and 5) no Att-SNARC was also observed when, after speeded detection, participants were asked to state whether the cue was an even or odd number, ie, Parity Judgement. Finally, in Experiment 3 from the first series of experiments the Att-SNARC was found when, following speeded detection of lateral targets, participants were asked to judge whether the cue was higher or lower than 5, ie, Magnitude Comparison. Nonetheless, this finding was not replicated in a control retest experiment in the second series of experiments (Experiment 6). It is worth noting that in a previous study run in a small sample of eight participants, Casarotti et al. (2007) used a temporal order judgement task with lateral targets preceded by central digit cues and found number-related shifts of attention when participants were asked to compare the magnitude of the cues with a central magnitude reference, ie, 5. As an example, when presented with two simultaneous targets, one to the left and one to the right of fixation, participants judged the target on the left side to occur before that on the right side when targets were preceded by a small magnitude digit cue, eg, 1 . Unfortunately, as in the case of the previously quoted ERP study by Schuller et al. (2015), the use of left/right codes both in temporal order judgements and in the selection of the left/right key press that were used to provide the same judgements precludes to conclude whether the attentional effects observed by Casarotti et al. (2007) are due to magnitude processing or to the concomitant use of spatial codes in the classification of target side and in the selection of corresponding motor responses.

In a more recent study (Fattorini et al., 2015b), we jointly addressed the reassessment of the strength and reliability of the Att-SNARC and investigated whether interindividual variations of its strength are correlated with equivalent variations 
in the strength of the PJ- and MC-SNARC effects. To this aim in a first experiment we administered the Att-SNARC and the PJ- and MC-SNARC to a large sample of 60 participants. The results were straightforward: no Att-SNARC was found whereas significant and reliable SNARC effects were observed in the same participants. In addition to this, no correlation between the Att-SNARC and the PJ- or MC-SNARC effects were observed, both in the whole sample of participants and when participants were divided in the subsamples of those showing a conventional or a reversed Att-SNARC. In a second study, in order to directly test whether central numerical cues putatively triggering lateral shifts of attention can directly modulate the SNARC effect, we merged the Att-SNARC and the MC-SNARC tasks in a single one. At the beginning of each trial, a central numerical cue was presented. The cue was followed by a numerical target that appeared in one of the two lateral positions used in the Att-SNARC task. Participants were asked to decide as fast as possible whether targets were smaller or larger than 5. Decisions were taken by choosing between an upper or a lower key, ie, between keys that were placed in a orthogonal direction with respect to target positions. We found a conventional MC-SNARC effect that was not modulated by the magnitude of central cues. In addition the magnitude of central cues did not modulate RTs to left-side or right-side targets, demonstrating the absence of the Att-SNARC. The results of these first two experiments led us to conclude, in agreement with Zanolie and Pecher (2014) and van Dijck et al. (2014), that the mere perception of numbers does not cause shifts of attention. In addition our study showed that, whenever present, shifts of attention related to mere number perception play no role in the genesis of the SNARC effect. In a third study, we investigated whether the conceptual association between number magnitude and left/right spatial codes can engender a stable link between number and space coding. This idea was inspired by the observation that in conventional SNARC tasks, left/right spatial codes are used to select motor responses and through this selection they are associated to number magnitudes. With this in mind, we wished to test whether a direct, ie, not mediated by response selection, and explicit association between number magnitudes and left/right spatial codes can make central numerical cues capable of inducing lateral shifts of spatial attention. To this aim we administered a conventional Att-SNARC in which following primary target detection through unimanual keypress responses, participants were asked to classify verbally whether on conventional rulers the numerical cue is placed to the left or to the right of the number 5 . In this case, we observed a significant Att-SNARC. Nonetheless, at variance with SNARC tasks, this effect was not fully reliable on split-half testing. All together these results led us to conclude that numbers have no inherent left-to-right organization and that this organization is adopted only when left/right spatial codes are used for response selection or, based on task instructions, are explicitly associated to number magnitudes: nonetheless, response-related factors seem to produce more stable spatial representations of numbers as compared to purely conceptual ones. 


\subsection{THE PRESENT STUDY: THE INFLUENCE OF TASK DEMANDS AND THE SET-SIZE OF NUMERICAL CUES ON THE ATTENTIONAL SNARC EFFECT}

The recent study by Zanolie and Pecher (2014) showed that the type of cognitive processing assigned to central numerical cues can influence the presence of the Att-SNARC effect. In their Experiment 3, these authors pointed out a significant Att-SNARC when, rather than merely and passively perceiving numerical cues, participants were required to process their magnitude and decide whether they were higher or lower than 5. Though this finding was not replicated in the Experiment 6 of the same study, it suggests that the active processing of specific number features might create a temporary and task-dependent link between number and spatial attentional mechanisms. Therefore, the first aim of the present study was to contrast the Att-SNARC effect observed with passive perception of numerical cues (Experiments 1a and 1b) with that observed when participants are asked to perform different types of active processing of the same numerical cues. In the second and third experiment of our study, we assessed the influence of two different types of spatial processing of numerical cues on the strength of the Att-SNARC. In the first case, we asked participants to imagine numerical cues as being placed on a conventional horizontal ruler, ie, Imagery Att-SNARC (Experiments $2 \mathrm{a}$ and $2 \mathrm{~b}$ ). This manipulation was inspired by the classical observations of introspective number forms in synestheses that we have summarized in Section 1. We wondered whether in normal unselected participants the active imaginative recall of a ruler might engender the number-space interaction. In the second case (Experiments 3a and 3b; Spatial Att-SNARC), we explored whether the explicit conceptual association of left/right spatial codes with number magnitudes is a relevant determinant of the Att-SNARC. This manipulation was inspired by the findings reported by Gevers et al. (2010) that verbal spatial coding has a relevant influence on the conventional SNARC effect. Gevers et al. (2010) showed that the SNARC effect is present also when participants are merely asked to make an association between verbal "left" or "right" responses and the parity of a centrally presented target number. In the same vein, here we tested whether asking participants to state whether the numerical cue presented in each trial is positioned to the "left" or the "right" of the number " 5 " on an imagined ruler could engender a significant and reliable Att-SNARC. Note that this Spatial Att-SNARC differs from the Imagery Att-SNARC of experiment 2, because in the former case an explicit numerical reference is given inside the mental image. Therefore, in this third experiment we wished to replicate the original results of the third experiment reported in Fattorini et al. (2015b). Finally, in the fourth experiment (Experiments 4a and 4b) we verified whether a consistent Att-SNARC is generated when participant must perform Magnitude Comparison judgments of central digit cues using purely quantitative codes and no explicit left/right spatial codes, ie, Magnitude Comparison Att-SNARC.

In all experiments another important variable was manipulated. To our knowledge, virtually in all investigations of the Att-SNARC only four-digit cues $(1,2$, 
8, and 9) are typically considered. This is different from what usually happens in SNARC tasks, where a continuous representation of the typically larger set of eight items (1-9 excluding 5) is usually made evident in Magnitude Comparison SNARC by the presence of a clear and continuous distance effect in reaction times (Moyer and Landauer, 1967). Therefore, here we wished to investigate whether using a larger set of digit cues modifies or provides important qualifications of the Att-SNARC effect. As an example, in a recent paper we advanced the hypothesis that the strength of number-related shifts of attention could be a continuous function of the numerical distance between the digit cue and a numerical reference on the MNL (Rossetti et al., 2011). In this case, if one takes as reference the number 5, the digit cue 9 should produce larger attentional advantages in the detection of target in the right side of space as compared with 8,8 larger advantages as compared with 7, and so on. With this aim, each of the variations of the Att-SNARC tasks considered in the present study was run twice: once using a small set of four numerical cues (1, 2, 8, and 9) and once using a larger set of eight numerical cues (1, 2, 3, 4, $6,7,8$, and 9).

\section{EXPERIMENT 1: ATTENTIONAL SNARC}

\subsection{EXPERIMENT 1A: FOUR-DIGIT CUES (1, 2, 8, AND 9)}

\subsubsection{Method}

Participants. Twenty healthy right-handed undergraduate students participated in the experiment ( 15 females, 5 males; mean age $=22.3$ years, $\mathrm{SD}=1.8$ years). All participants were recruited from the University "Sapienza" in Rome, had normal or corrected to normal vision and were blind to the aims of the study.

Apparatus. Participants were tested in a sound attenuated room with dim illumination. Stimuli were presented on a 15-in. color 6546 IBM monitor. An Intel Pentium 4 PC running E-Prime software (Schneider et al., 2002) controlled the presentation of stimuli and the recording of responses. Viewing distance from the screen was $57 \mathrm{~cm}$.

Attentional SNARC task. The experimental task was that proposed by Fischer et al. (2003). A central fixation cross $(0.4$ degree $\times 0.4$ degree $)$ was presented at the beginning of each trial together with two lateral boxes ( 1 degree $\times 1$ degree) for $500 \mathrm{~ms}$. One box was centered 5 degree to the left of central fixation and the other box 5 degree to the right of fixation. At the end of this $500 \mathrm{~ms}$ period, one out of fourdigit cues (ie, 1,2 , 8, or 9; size 0.8 degree $\times 0.6$ degree) appeared for $300 \mathrm{~ms}$ at central fixation. Following cue presentation, the central fixation cross reappeared and after a further delay of 500 or $750 \mathrm{~ms}$ (ie, CTI) a white asterisk-target $(0.5$ degree $\times 0.5$ degree) was randomly presented inside one of the two lateral boxes for $300 \mathrm{~ms}$. The two CTIs (ie, 500 and $750 \mathrm{~ms}$ ) were those at which a significant Att-SNARC effect was originally reported by Fischer et al. (2003; see also Fattorini et al., 2015b). Participants were asked to press the space bar with their right index finger as quick as possible in response to the target. An intertrial interval of 
2200 ms was interposed between the response and the start of the ensuing trial. Before testing, participants were instructed to maintain gaze at central fixation during task performance and were informed that digit cues presented at fixation were irrelevant to target detection. Digit cues were not predictive of target location. The task consisted of 384 experimental trials (96 repetitions for each digit cue) and 96 catch trials with no target presentation. Trials were administered in four consecutive blocks, separated by a short self-paced pause. A training session including 16 experimental trials ( 1 trial $\times$ digit $\times$ CTI $\times$ target side $)$ was administered ahead of experimental blocks.

Data filtering. Responses to catch trials (false alarms), trials in which no response was made (misses) and trials in which RTs were above or below two standard deviations from the mean of the experimental condition in which the trial occurred were considered outliers and were not included in the statistical analyses.

\subsubsection{Results}

Attentional SNARC effect. $4.2 \%$ of trials were discarded from the analyses. To evaluate the presence of the Att-SNARC individual average RTs were first entered in a CTI $(500,750 \mathrm{~ms}) \times$ Digit-Magnitude (small, large) $\times$ Target-Side (left, right) repeated measures ANOVA. No significant main effects or interactions were found. Crucially, neither the Digit-Magnitude $\times$ Target-Side nor the CTI $\times$ DigitMagnitude $\times$ Target-Side interactions that qualify the Att-SNARC effect were significant $\left[F(1,19)=3.52, p=0.082, \eta^{2}=0.20 ; F<1\right.$, respectively; note that the $p=0.082$ Digit-Magnitude $\times$ Target-Side interaction highlights a bias toward reversed Att-SNARC with left-side targets: large cue $=346 \mathrm{~ms}$ vs small cue $=356 \mathrm{~ms}]$. The same null results were observed in a CTI $(500,750 \mathrm{~ms}) \times$ Digit Cue $(1,2,8$, and $9) \times$ Target-Side (left, right) ANOVA. Also in this case, the Digit Cue $\times$ Target-Side and the CTI $\times$ Digit Cue $\times$ Target-Side interactions were not significant $[F(3,57)=$ $1.49, p=0.23, \eta^{2}=0.09 ; \quad F(3,69)=1.11, p=0.36, \eta^{2}=0.07$, respectively]. See Fig. 1.

The Att-SNARC effect was also evaluated through a regression analysis (Lorch and Myers, 1990). For each participant, average RTs recorded on trials with left- and right-side targets were computed for each digit cue separately. Differential RTs (dRTs) were then computed by subtracting the average RTs recorded in trials with left-side targets from average RTs recorded in trials with right-side targets. According to the Att-SNARC, small digits should elicit faster response to left targets, thus producing positive dRTs, whereas large digits should elicit faster response to right targets, thus producing negative dRTs. Based on dRTs, we calculated individual linear regression equations (ie, individual slopes) using digit cue magnitude as the predictor variable. In this case, a negative regression slope should qualify the AttSNARC. To assess whether the average group regression slope was different from zero we entered individual regression slopes in one-sample $t$-test. The average group slope was not different from zero (Fig. 1) both when the two CTI conditions were collapsed and when the two CTI conditions were considered separately, which demonstrates the absence of the Att-SNARC effect [Collapsed-CTIs: $t(19)=1.75$, 
A
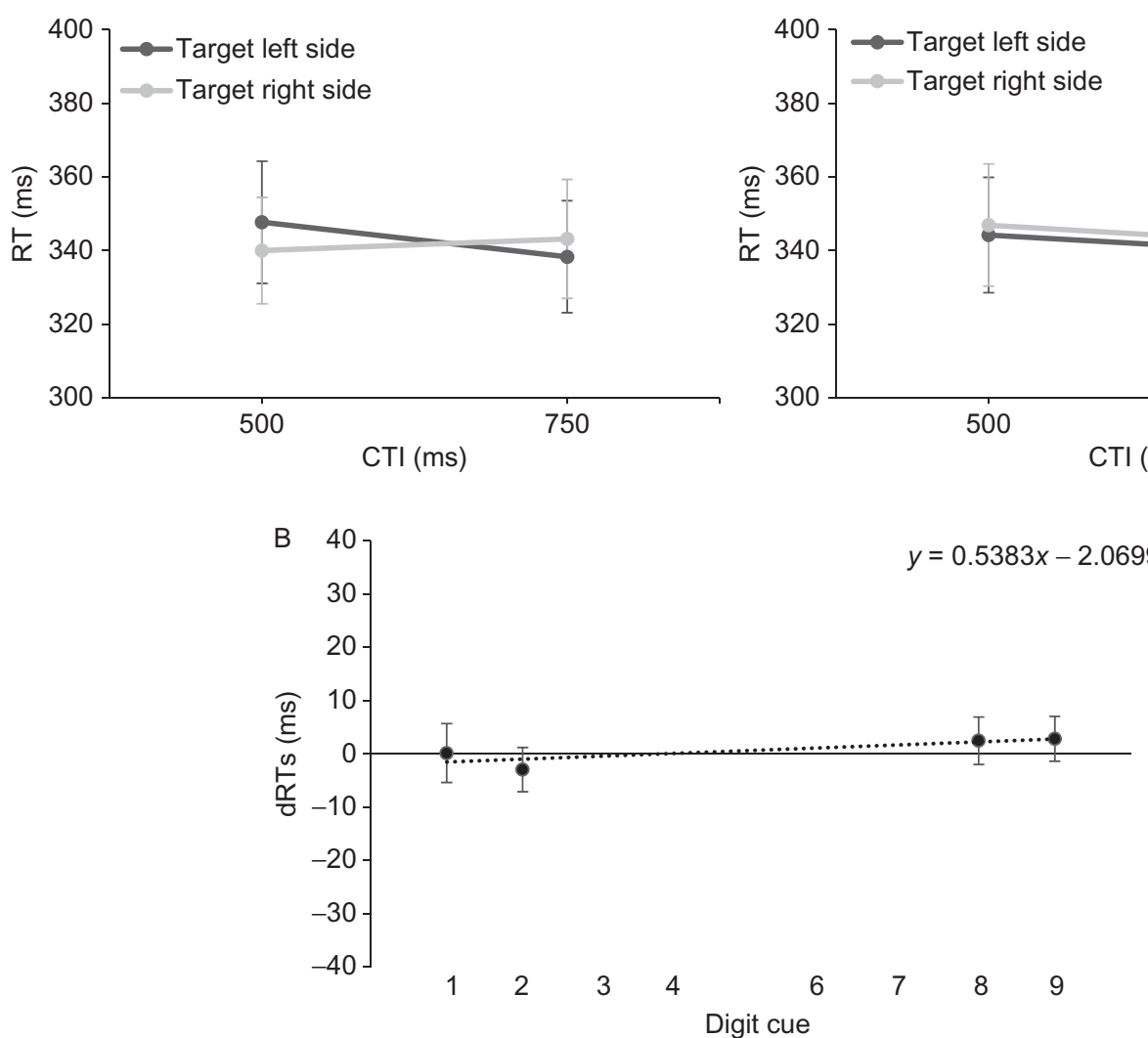

High digit cue $(8,9)$

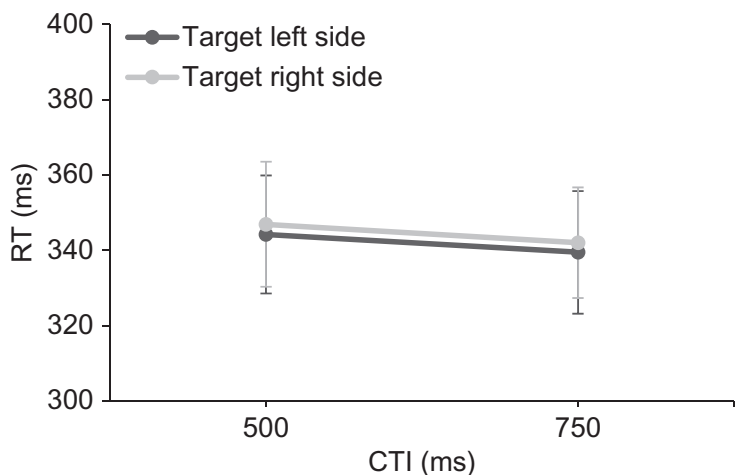

$\mathrm{CTI}(\mathrm{ms})$

\section{FIG. 1}

Experiment 1a: Att-SNARC task with four-digit cues. (A) Average RTs (with s.e.) to targets presented in the left and right side of space plotted as function of the magnitude of central digit cues, low (1 and 2) or high (8 and 9), and cue-target intervals. (B) Slope describing the difference between RTs to targets in the right side of space minus targets in the left side of space, plotted as a function of the magnitude of central digit cues. 
$p=0.10$, Slope $=1.01, \mathrm{SD}=2.43 ; 500$ ms-CTI: $t(19)=0.97, p=0.35$, Slope $=0.90$, $\mathrm{SD}=3.62 ; 750 \mathrm{~ms}-\mathrm{CTI}: t(19)=1.51, p=0.15$, Slope $=1.29, \mathrm{SD}=3.32$ ].

\subsection{EXPERIMENT 1B: EIGHT-DIGIT CUES (1, 2, 3, 4, 6, 7, 8, AND 9)}

\subsubsection{Method}

Participants. Twenty healthy right-handed undergraduate students (16 females, 4 males; mean age $=21.8$ years, $\mathrm{SD}=1.7$ years).

Attentional SNARC task. The task was the same Attentional SNARC task of Experiment 1a, except that all numbers between 1 and 9 (digit 5 excluded) were administered as digit cues. The task consisted of 720 experimental trials (ie, 80 repetitions for each digit cue) and 144 catch trials with no target presentation. A training session with 18 trials (ie, 1 repetition for each cue-target pairing) was administered before the experimental session.

\subsubsection{Results}

Attentional SNARC effect. $5.1 \%$ of trials were discarded from the analyses. The CTI $(500,750 \mathrm{~ms}) \times$ Digit-Magnitude (small, large) $\times$ Target-Side (left, right) ANOVA highlighted a significant Digit-Magnitude $\times$ Target-Side interaction $\left[F(1,19)=6.51, p=0.018, \eta^{2}=0.22\right]$ showing a reversed Att-SNARC effect. In the second CTI $(500,750 \mathrm{~ms}) \times$ Digit Cue $(1,2,3,4,6,7,8$, and 9) $\times$ Target-Side (left, right) ANOVA, the two interactions of interest qualifying the presence of the Att-SNARC did not reach statistical significance $[F(7,133)=1.71, p=0.1$, $\eta^{2}=0.07$ for Digit Cue $\times$ Target-Side; and $F<1$ for $\mathrm{CTI} \times$ Digit Cue $\times$ TargetSide]. See Fig. 2.

In order to assess the hypothesis that the strength of number-related shifts of attention is affected by the numerical distance between the digit cue and the numerical reference (ie, 5), we computed individual mean RTs for each digit cue separately and tested whether the Att-SNARC effect changed as a function of the numerical distance between the digit cue and the reference (distance $1=$ digits 4 and 6 ; distance $2=$ digits 3 and 7 ; distance $3=$ digits 2 and 8 ; distance $4=\operatorname{digits} 1$ and 9). A Digit Cue/Target-Side condition (Congruent, Incongruent) $\times$ Distance to reference $(1,2,3$, and 4) ANOVA highlighted no main Distance effect $[F(3,57)=0.6$, $\left.p=0.61, \eta^{2}=0.02\right]$ showing that the RTs were not modulated by the numerical distance between digit cues and the central reference. The interaction between Digit cue/Target-Side condition and Distance was not significant $[F(3,57)=0.03$, $\left.p=0.80, \eta^{2}=0.01\right]$.

The regression analyses (Fig. 2) confirmed the absence of the conventional AttSNARC and showed a reversed Att-SNARC at $750 \mathrm{~ms}-\mathrm{CTI}$ and when the two CTI conditions were collapsed [Collapsed-CTIs: $t(19)=2.49, p=0.02$, Slope $=0.97$, $\mathrm{SD}=1.89 ; 500 \mathrm{~ms}-\mathrm{CTI}: t(19)=1.36, p=0.19$, Slope $=0.68, \mathrm{SD}=2.48 ; 750 \mathrm{~ms}-$ CTI: $t(19)=2.14, p=0.043$, Slope $=1.25, \mathrm{SD}=2.86]$. 
A

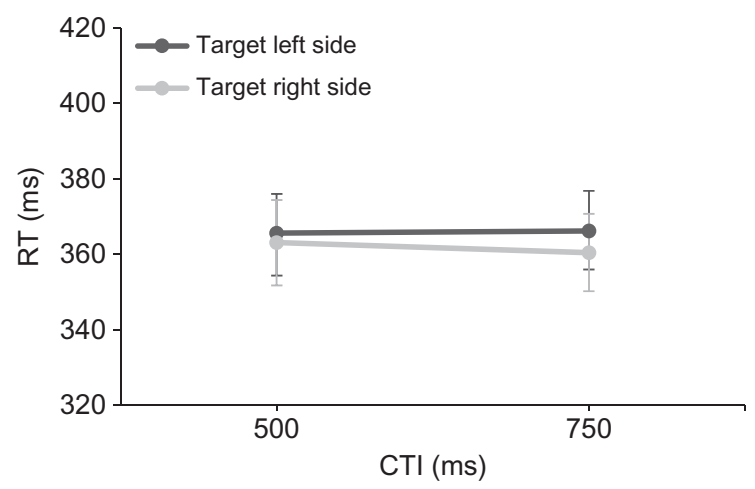

$\mathrm{B}$

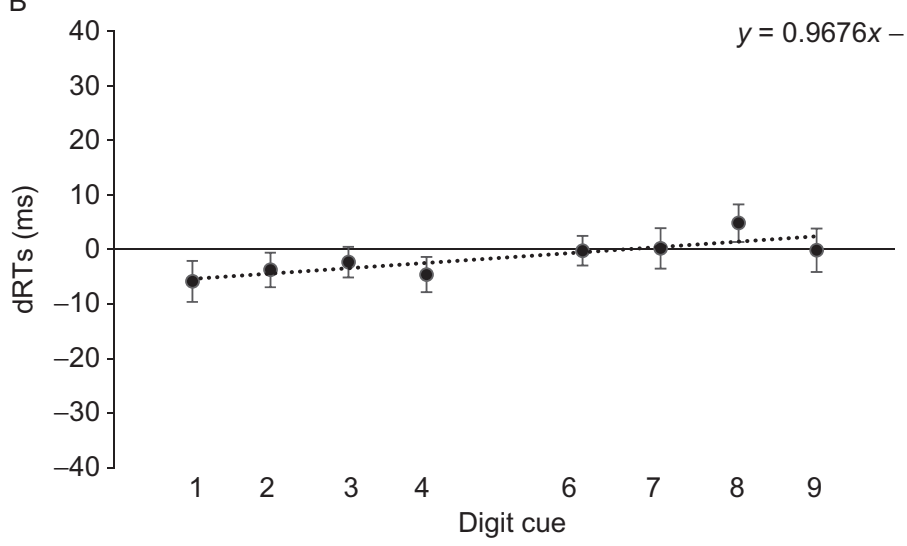

High digit cue $(6,7,8,9)$

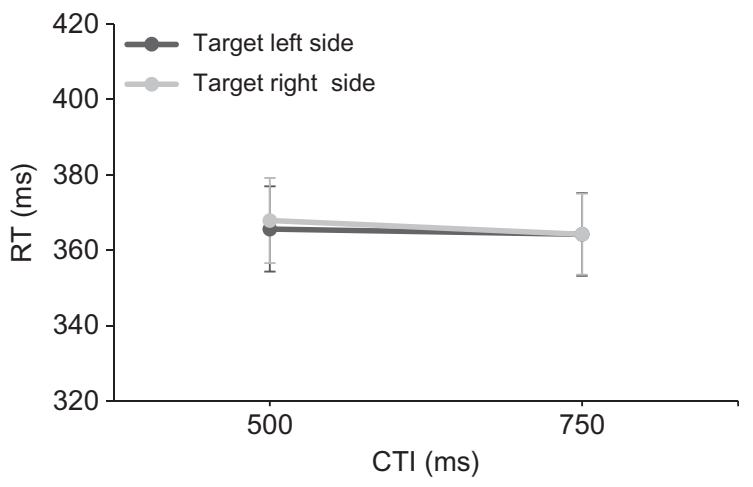

\section{FIG. 2}

Experiment 1b: Att-SNARC task with eight-digit cues. (A) Average RTs (with s.e.) to targets presented in the left and right side of space plotted as function of the magnitude of central digit cues, low (1, 2, 3, and 4) or high (6, 7, 8, and 9), and cue-target intervals. (B) Slope describing the difference between RTs to targets in the right side of space minus targets in the left side of space, plotted as function of the magnitude of central digit cues. 


\subsubsection{Comparison of the strength of the Att-SNARC between the four- and eight-digit cues conditions}

A $t$-test for independent samples run on individual slopes showed that the AttSNARC was equivalent between the four- and eight-digit cues conditions $(t(38)=0.19, p=0.85)$.

\section{EXPERIMENT 2: IMAGERY ATTENTIONAL SNARC}

\subsection{EXPERIMENT 2A: FOUR-DIGIT CUES (1, 2, 8, AND 9)}

\subsubsection{Method}

Participants. Twenty healthy right-handed undergraduate students (17 females, 3 males; mean age $=23.3$ years, $\mathrm{SD}=2.2$ years).

Imagery Attentional SNARC task. The Imagery Attentional SNARC task was like the Attentional SNARC task of Experiment 1a, except that participants were asked to imagine digit cues as being positioned on a left-to-right oriented spatial ruler.

\subsubsection{Results}

Imagery Attentional SNARC effect. 3.9\% of trials were discarded from the analyses. Individual average RTs were first entered in a CTI $(500,750 \mathrm{~ms}) \times$ Digit-Magnitude (small, large) $\times$ Target-Side (left, right) ANOVA. The Digit-Magnitude $\times$ TargetSide and the CTI $\times$ Digit-Magnitude $\times$ Target-Side interactions were not significant $\left[F(1,19)=1.91, p=0.19, \eta^{2}=0.11 ; F(1,19)=2.27, p=0.15, \eta^{2}=0.13\right.$, respectively], revealing the absence of the Att-SNARC effect (see Fig. 3). A second CTI $(500,750 \mathrm{~ms}) \times$ Digit Cue $(1,2,8$, and 9) $\times$ Target-Side (left, right) ANOVA revealed a significant $\mathrm{CTI} \times$ Digit Cue $\times$ Target-Side interaction $[F(3,57)=3.93$, $\left.p=0.014, \eta^{2}=0.21\right]$. This interaction was qualified by a reversed Att-SNARC effect for digit $1\left[F(3,57)=4.24, p=0.01, \eta^{2}=0.22\right.$; left target $=349 \mathrm{~ms}$ vs right target $=337 \mathrm{~ms}]$.

Regression analyses (see Fig. 4) confirmed the absence of the conventional AttSNARC and showed a reversed Att-SNARC in the $500 \mathrm{~ms}-\mathrm{CTI}$ condition [Collapsed-CTIs: $t(19)=1.39, p=0.18$, Slope $=0.54, \mathrm{SD}=1.55 ; 500 \mathrm{~ms}-\mathrm{CTI}$ : $t(19)=2.71, p=0.016$, Slope $=1.58, \mathrm{SD}=2.33 ; 750 \mathrm{~ms}-\mathrm{CTI}: t(19)=-0.61$, $p=0.55$, Slope $=-0.5, \mathrm{SD}=3.28]$.

\subsection{EXPERIMENT 2B: EIGHT-DIGIT CUES (1, 2, 3, 4, 6, 7, 8, AND 9)}

\subsubsection{Method}

Participants. Twenty healthy right-handed undergraduate students (14 females, 6 males; mean age $=22.7$ years, $\mathrm{SD}=2.4$ years).

Imagery Attentional SNARC task. The Imagery Attentional SNARC task was the same of Experiment 2a. Stimuli, types of trials, number of trials and training procedure were as in Experiment $1 \mathrm{~b}$. 


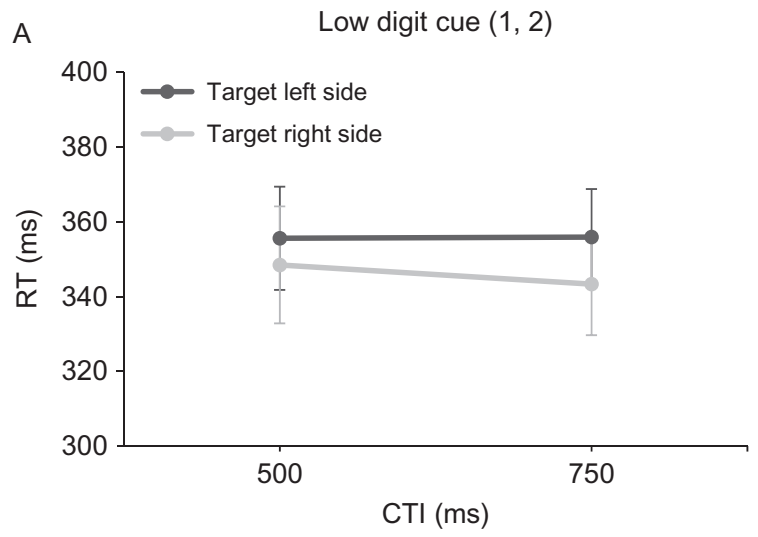

B

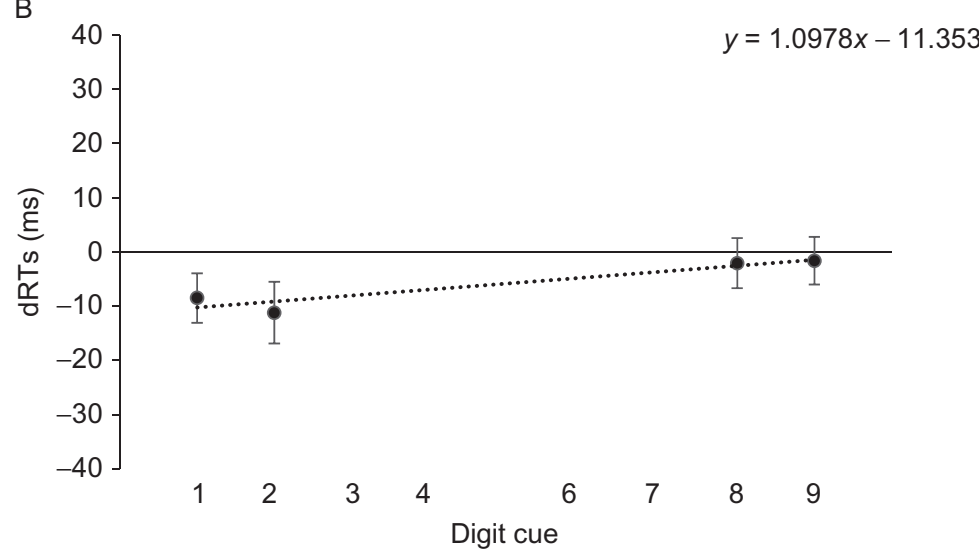

High digit cue $(8,9)$

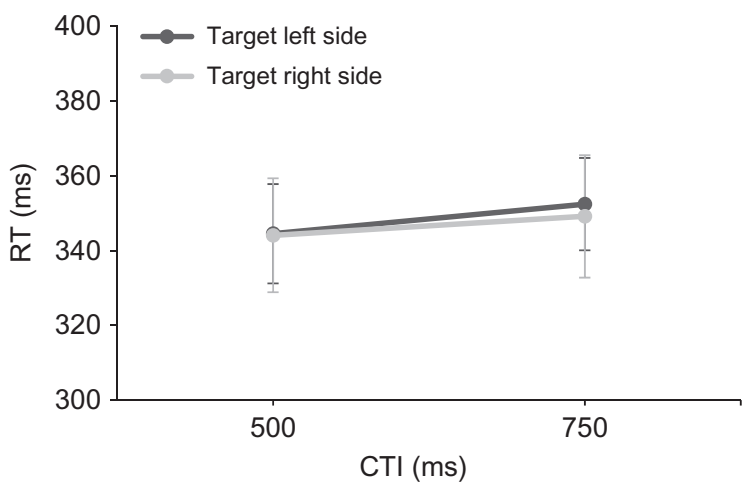

\section{FIG. 3}

Experiment 2a: Imagery Att-SNARC task with four-digit cues. (A) Average RTs (with s.e.) to targets presented in the left and right side of space plotted as function of the magnitude of central digit cues, low (1 and 2) or high (8 and 9), and cue-target intervals. (B) Slope describing the difference between RTs to targets in the right side of space minus targets in the left side of space, plotted as function of the magnitude of central digit cues. 

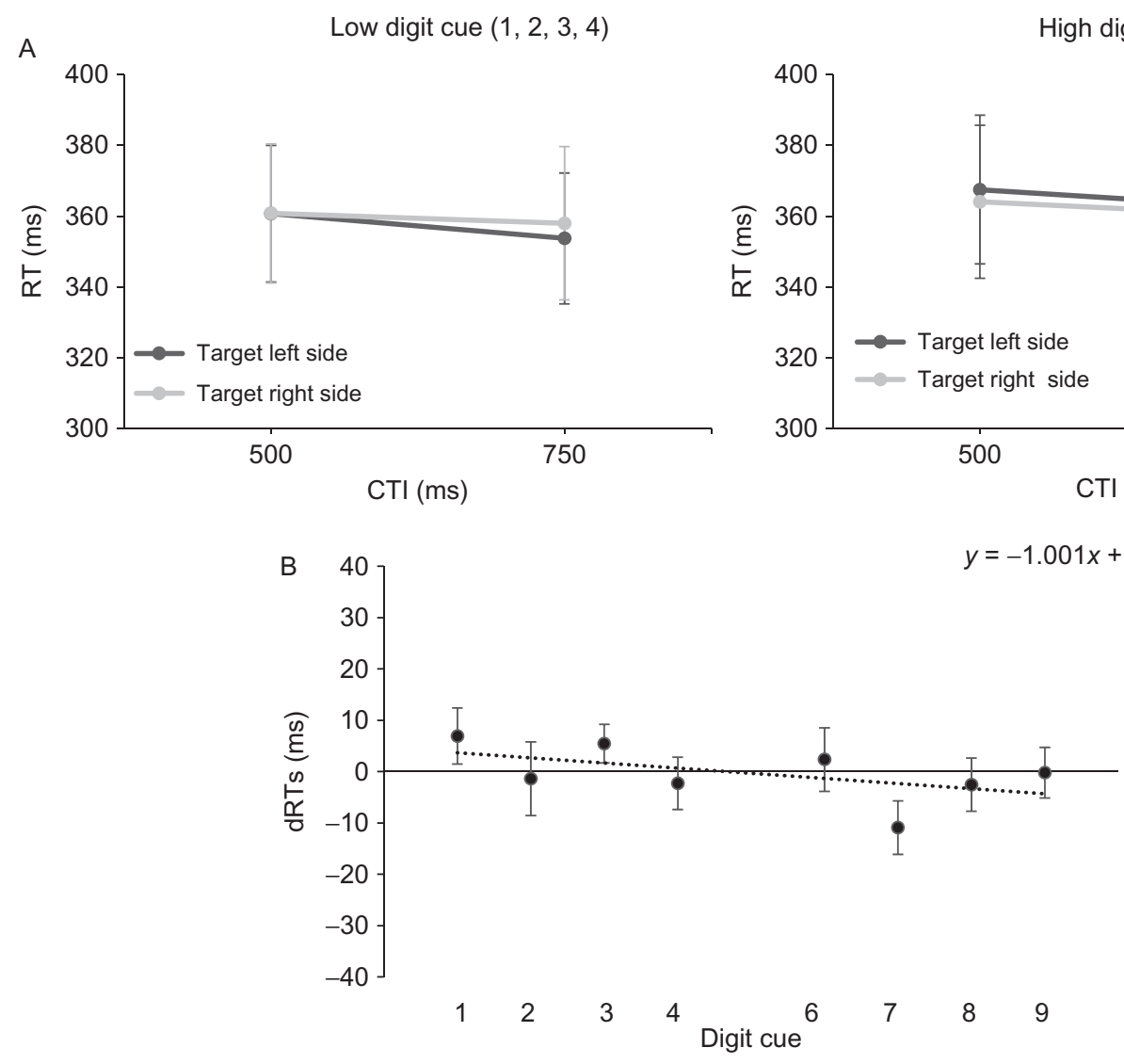

High digit cue $(6,7,8,9)$

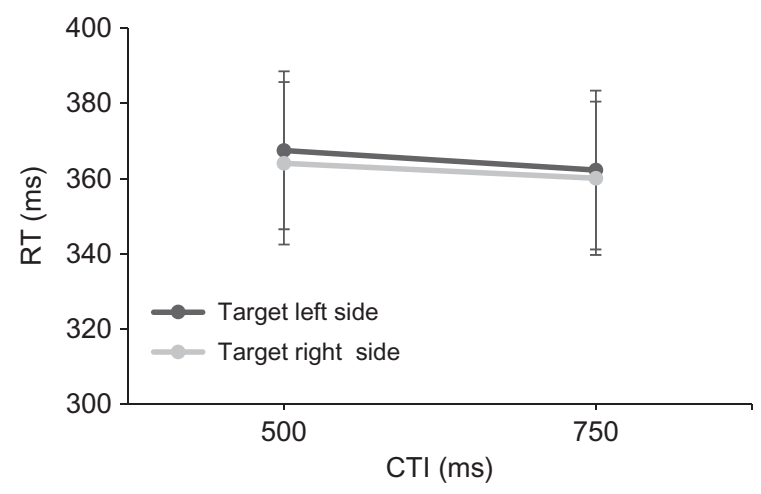

\section{FIG. 4}

Experiment 2b: Imagery Att-SNARC task with eight-digit cues. (A) Average RTs (with s.e.) to targets presented in the left and right side of space plotted as function of the magnitude of central digit cues, low (1, 2, 3, and 4) or high (6, 7, 8, and 9), and cue-target intervals. (B) Slope describing the difference between RTs to targets in the right side of space minus targets in the left side of space, plotted as function of the magnitude of central digit cues. 


\subsubsection{Results}

Imagery Attentional SNARC effect. 5.7\% of trials were discarded from the analyses. CTI $(500,750 \mathrm{~ms}) \times$ Digit-Magnitude (small, large) $\times$ Target-Side (left, right) and CTI $(500,750 \mathrm{~ms}) \times$ Digit Cue $(1,2,3,4,6,7,8$, and 9) $\times$ Target-Side (left, right) ANOVA revealed no significant Att-SNARC [First ANOVA: Digit-Magnitude $\times$ Target-Side, $F(1,19)=1.51, p=0.24, \eta^{2}=0.10$; CTI $\times$ DigitMagnitude $\times$ Target-Side, $F<1$; Second ANOVA Digit-Magnitude $\times$ Target-Side, $F \quad(7, \quad 133)=1.29, \quad p=0.25, \quad \eta^{2}=0.08 ; \quad$ CTI $\times$ Digit-Magnitude $\times$ Target-Side $F<1$ ]. See Fig. 4.

The Digit Cue/Target-Side condition (Congruent, Incongruent) $\times$ Distance to reference $(1,2,3$, and 4) ANOVA highlighted a main distance effect that approached significance $\left[F(3,57)=2.06, p=0.12, \eta^{2}=0.12\right]$ showing that the RTs were not modulated by the numerical distance between digit cues and central reference. The interaction between Digit Cue/Target-Side condition and distance did not approach significance $\left[F(3,57)=1.69, p=0.19, \eta^{2}=0.1\right]$.

The regression analyses confirmed the absence of the Att-SNARC effect [Collapsed-CTIs: $t(19)=-1.15, p=0.27$, Slope $=-1, \mathrm{SD}=3.35 ; 500 \mathrm{~ms}-\mathrm{CTI}$ : $t(19)=-1.46, p=0.17$, Slope $=-0.92, \mathrm{SD}=2.44 ; 750$ ms-CTI: $t(19)=-0.81$, $p=0.44$, Slope $=-1.1, \mathrm{SD}=5.24]$. See Fig. 4 .

\subsubsection{Comparison of the strength of the Att-SNARC between the four- and eight-digit cues conditions}

The Imagery Att-SNARC was not significantly different between the four- and eightdigit cues conditions $(t(38)=1.71, p=0.1)$.

\section{EXPERIMENT 3: SPATIAL ATTENTIONAL SNARC}

\subsection{EXPERIMENT 3A: FOUR-DIGIT CUES (1, 2, 8, AND 9)}

\subsubsection{Method}

Participants. Twenty-four healthy right-handed undergraduate students (18 females, 6 males; mean age $=23.3$ years, $\mathrm{SD}=2.4$ years).

Spatial Attentional SNARC task. The task was identical to the Attentional SNARC task of Experiment 1a, except that in each trial a verbal-spatial judgement on numerical cues was required after speeded target detection, ie, "is the number positioned to the left or to the right of number 5 on conventional rulers?" Verbal responses were recorded through a microphone for off-line scoring.

\subsubsection{Results}

Spatial Attentional SNARC effect. $4.9 \%$ of trials were discarded from the analyses. No error was virtually observed in the left/right classification of digit cues (ie, error rate $<0.2 \%$ ). Individual mean RTs were first entered in a CTI (500, $750 \mathrm{~ms}) \times$ Digit-Magnitude (small, large) $\times$ Target-Side (left, right) repeated measures ANOVA. The interaction between Digit-Magnitude and Target-Side was 
significant $\left[F(1,23)=41.8, p<0.001, \eta^{2}=0.65\right]$ and highlighted the presence of the Att-SNARC effect. Post hoc tests showed that the Att-SNARC was significant both for small and large digits [small digit-magnitude: left target $=338 \mathrm{~ms}$ vs right target $=352 \mathrm{~ms}, p<0.001$; large digit-magnitude: left target $=351 \mathrm{~ms}$ vs right target $=333 \mathrm{~ms}, p<0.001]$. A second CTI $(500,750 \mathrm{~ms}) \times$ Digit Cue $(1,2,8$, and $9) \times$ Target-Side (left, right) ANOVA confirmed the presence of the Att-SNARC effect [Digit Cue $\times$ Target-Side interaction: $F(3,69)=21.32, \quad p<0.001$, $\left.\eta^{2}=0.48\right]$ and post hoc tests showed that the Att-SNARC was significant for each digit cue [1: left target $=336 \mathrm{~ms}$ vs right target $=354 \mathrm{~ms}, p<0.001 ; 2$ : left target $=340 \mathrm{~ms}$ vs right target $=351 \mathrm{~ms}, p=0.015$; 8 : left target $=349 \mathrm{~ms}$ vs right target $=334 \mathrm{~ms}, p<0.01$; 9: left target $=353 \mathrm{~ms}$ vs right target $=331 \mathrm{~ms}$, $p<0.001]$. See Fig. 5 .

Regression analyses confirmed the Att-SNARC effect for each CTI condition [Collapsed-CTIs: $t(23)=-6.7, p<0.001$, Slope $=-4.8, \mathrm{SD}=3.5 ; 500 \mathrm{~ms}-\mathrm{CTI}$ : $t(23)=-5.2, p<0.001$, Slope $=-4.5, \mathrm{SD}=4.3 ; 750 \mathrm{~ms}-\mathrm{CTI}: t \quad(23)=-4.8$, $p<0.001$, Slope $=-5.1, \mathrm{SD}=5.1]$. See Fig. 5 .

Finally, we assessed the reliability of the Att-SNARC effect using the split-half method with Spearman-Brown correction. Individual mean RTs for the first and second half of the task were calculated separately for CTI conditions (ie, $500 \mathrm{~ms}-\mathrm{CTI}$, 750 ms-CTI, and Collapsed-CTIs). Then, the individual dRTs between right and left targets were computed and the corresponding linear regression slopes were computed using each digit cue as predictor variable. Corrected Spearman-Brown correlations between the individual slopes of the first and second half of the task were computed. Split-half testing showed that the Att-SNARC effect approached reliability at $750 \mathrm{~ms}-\mathrm{CTI}\left[r_{1,2}=0.36\right.$, Spearman-Brown correction $\left.=0.53, p=0.082\right]$ and resulted not reliable at $500 \mathrm{~ms}-\mathrm{CTI}\left[r_{1,2}=-0.12\right.$, Spearman-Brown correction $=-0.29, p=0.55]$ or when the two CTIs were collapsed $\left[r_{1,2}=0.07\right.$, Spearman-Brown correction $=0.13, p=0.73]$.

\subsection{EXPERIMENT 3B: EIGHT-DIGIT CUES (1, 2, 3, 4, 6, 7, 8, AND 9)}

\subsubsection{Method}

Participants. Twenty-four healthy right-handed undergraduate students (17 females, 7 males; mean age $=22.8$ years, $\mathrm{SD}=1.9$ years).

Spatial Attentional SNARC task. The Spatial Attentional SNARC task was the same of Experiment 3a. Stimuli, types of trials, number of trials, and training procedure were as in Experiment $1 \mathrm{~b}$.

\subsubsection{Results}

Spatial Attentional SNARC effect. $6.1 \%$ of trials were discarded from the analyses. No error was virtually observed in the left/right classification of digit cues (ie, error rate $<0.4 \%)$. Individual mean RTs were first entered in a CTI $(500,750 \mathrm{~ms}) \times$ DigitMagnitude (small, large) $\times$ Target-Side (left, right) repeated measures ANOVA. The interaction between Digit-Magnitude and Target-Side resulted significant 
A

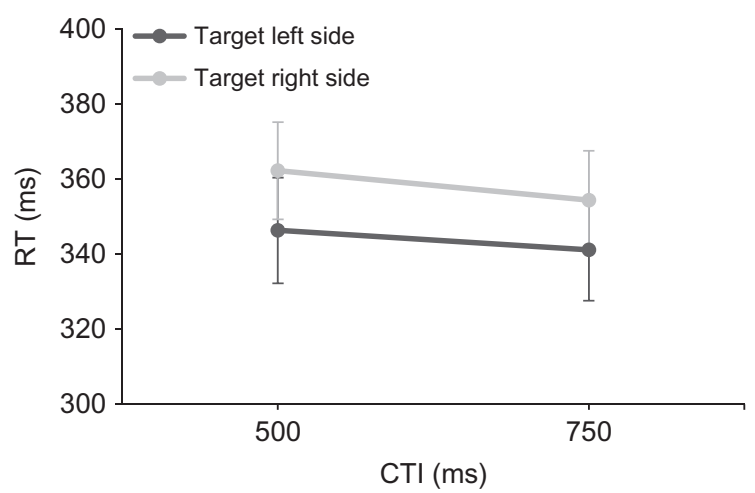

High digit cue $(8,9)$

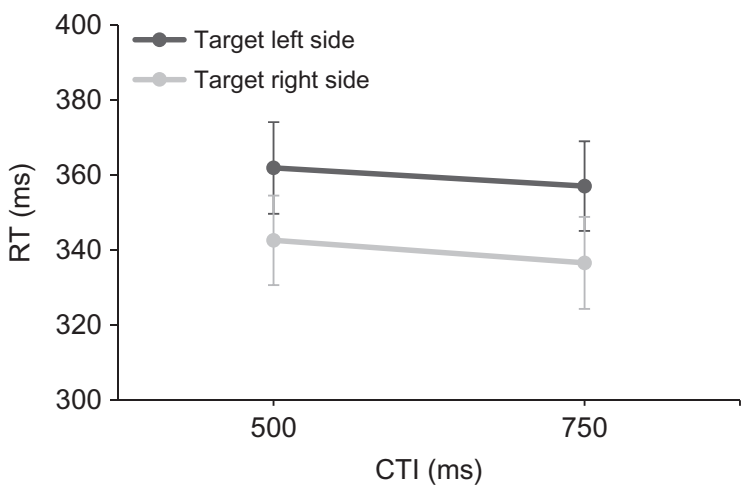

$y=-4.9692 x+22.211$

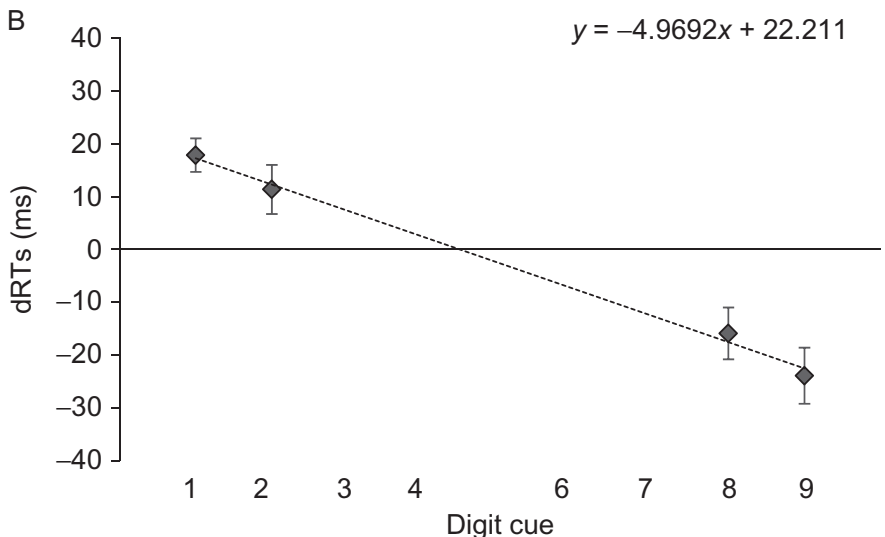

FIG. 5

Experiment 3a: Spatial Att-SNARC task with four-digit cues. (A) Average RTs (with s.e.) to targets presented in the left and right side of space plotted as function of the magnitude of central digit cues, low (1 and 2) or high (8 and 9), and cue-target intervals. (B) Slope describing the difference between RTs to targets in the right side of space minus targets in the left side of space, plotted as function of the magnitude of central digit cues. 
$\left[F(1,23)=7.59, p<0.05, \eta^{2}=0.31\right]$ and highlighted the presence of the AttSNARC effect. Post hoc tests showed that the Att-SNARC was only significant for large digits [small digit-magnitude: left target $=377 \mathrm{~ms}$ vs right target $=380 \mathrm{~ms}$, $p=0.49$; large digit-magnitude: left target $=386 \mathrm{~ms}$ vs right target $=372 \mathrm{~ms}$, $p<0.01]$. The second CTI $(500,750 \mathrm{~ms}) \times$ Digit Cue $(1,2,3,4,6,7,8$, and 9$) \times$ Target-Side (left, right) ANOVA confirmed the presence of the Att-SNARC effect [Digit Cue $\times$ Target-Side interaction: $F(7,161)=2.84, p<0.01, \eta^{2}=0.14$ ]. Post hoc tests showed that the Att-SNARC was significant for 6-, 7-, and 9-digit cues [6: left target $=388 \mathrm{~ms}$ vs right target $=373 \mathrm{~ms}, p=0.03 ; 7:$ left target $=394 \mathrm{~ms}$ vs right target $=370 \mathrm{~ms}, p=0.001$; and 9: left target $=380 \mathrm{~ms}$ vs right target $=360 \mathrm{~ms}$, $p=0.008$ ]. See Fig. 6 .

To test the influence of the numerical distance between the digit cues and the central spatial reference (ie, number 5) on number-related shifts of attention we ran a Digit Cue/Target-Side condition (Congruent, Incongruent) $\times$ Distance to reference $(1,2,3$, and 4) ANOVA. The main effect of Distance was significant $\left[F(3,57)=7.37, p<0.001, \eta^{2}=0.30\right]$ and showed that RTs increased progressively as an inverse function of the distance from the central reference. Post hoc tests were all significant $(p<0.01)$ except for the comparison between 3- and 4-unit distance $(p=0.27)$ and for that between 1 - and 2-unit distance $(p=0.89)$. The interaction between Digit Cue/Target-Side condition and Distance was not significant $[F(3,57)=$ $\left.0.91, p=0.44, \eta^{2}=0.05\right]$ showing that the numerical distance between the reference and the digit cues did not modulate the Spatial Att-SNARC effect.

Regression analyses showed the presence of a significant Att-SNARC effect at $750 \mathrm{~ms}-\mathrm{CTI}$ and when CTIs were collapsed [Collapsed-CTIs: $t(23)=-3.1$, $p<0.01$, Slope $=-2.9, \mathrm{SD}=4.1 ; 500 \mathrm{~ms}-\mathrm{CTI}: t(23)=-1.6, p=0.12$, Slope $=-2$, $\mathrm{SD}=5.2 ; 750$ ms-CTI: $t(23)=-2.7, p<0.05$, Slope $=-4.6, \mathrm{SD}=7.3]$. See Fig. 6 .

Finally, split-half testing showed that the Att-SNARC did not reach statistical reliability [Collapsed-CTIs: $r_{1,2}=-0.2$, Spearman-Brown correction $=-0.49$, $p=0.43$; 500 ms-CTI: $\quad r_{1,2}=-0.18$, Spearman-Brown $\quad$ correction $=-0.43$, $p=0.48 ; 750$ ms-CTI: $r_{1,2}=0.01$, Spearman-Brown correction $\left.=0.02, p=0.97\right]$.

\subsubsection{Comparison of the strength of the Att-SNARC between the four- and eight-digit cues conditions}

The Spatial Att-SNARC was not significantly different between the four- and eightdigit cues conditions $(t(38)=1.72, p=0.09)$.

\section{EXPERIMENT 4: MAGNITUDE ATTENTIONAL SNARC}

\subsection{EXPERIMENT 4A: FOUR-DIGIT CUES (1, 2, 8, AND 9)}

\subsubsection{Method}

Participants. Twenty-four healthy right-handed undergraduate students (18 females, 6 males; mean age $=23.1$ years, $\mathrm{SD}=2.3$ years). 
A

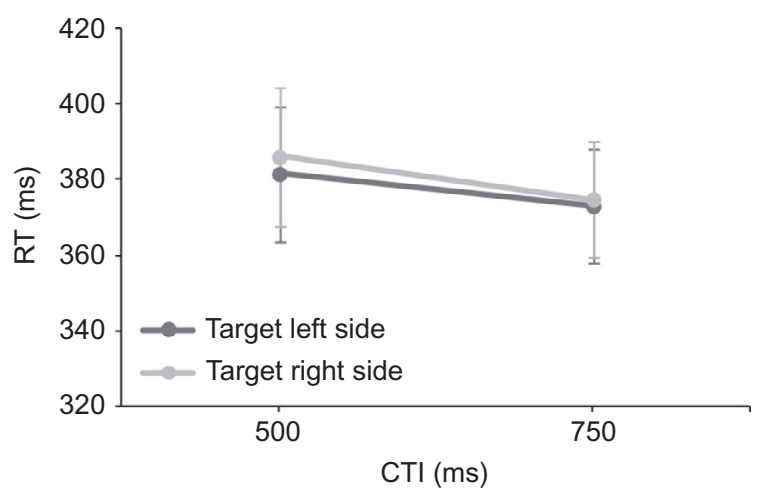

B

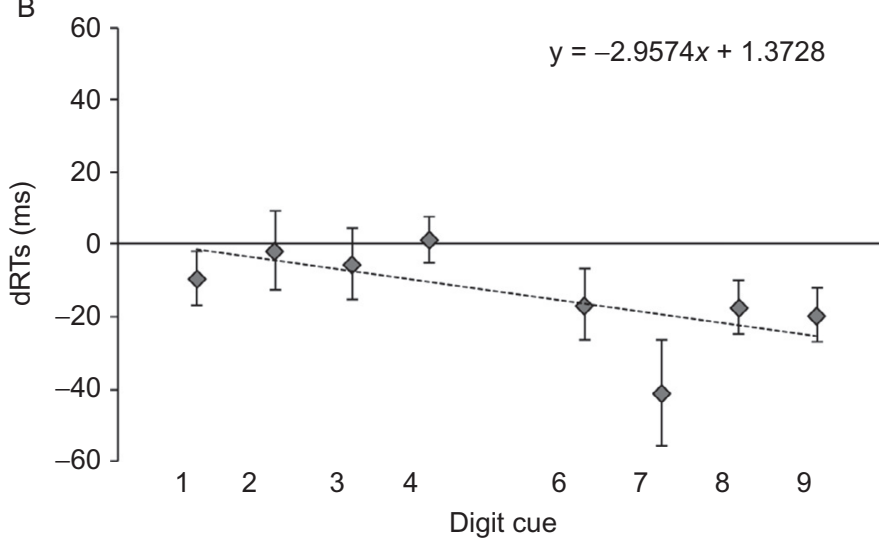

High digit cue $(6,7,8,9)$

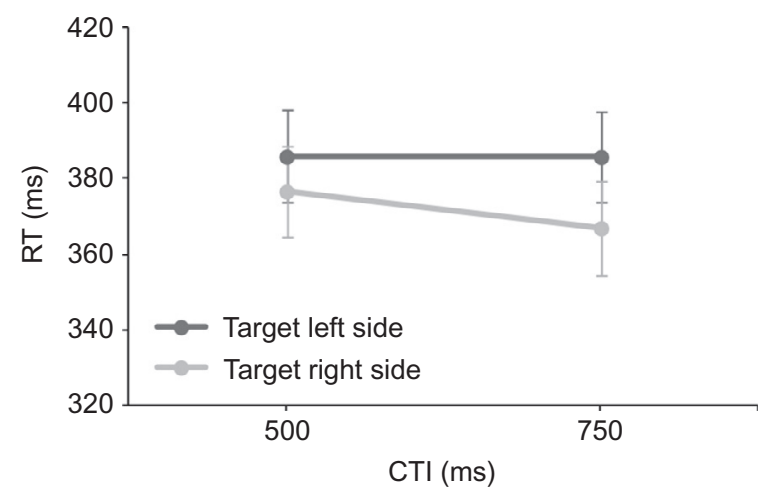

C

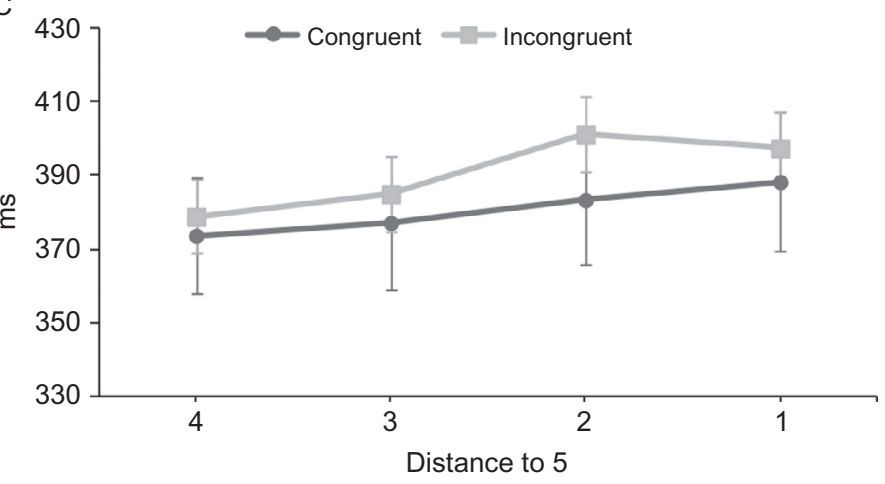

\section{FIG. 6}

Experiment 3b: Spatial Att-SNARC task with eight-digit cues. (A) Average RTs (with s.e.) to targets presented in the left and right side of space plotted as function of the magnitude of central digit cues, low (1, 2, 3, and 4) or high (6, 7, 8, and 9), and cue-target intervals. (B) Slope describing the difference between RTs to targets in the right side of space minus targets in the left side of space, plotted as function of the magnitude of central digit cues. (C) Reaction times in congruent and incongruent trials plotted as a function of the numerical distance (in units) between digit cues and the central mental spatial reference, ie, 5: a clear numerical distance effect is visible with faster reaction the larger the distance. 
Magnitude Attentional SNARC task. The Magnitude Attentional SNARC task was identical to the Spatial Attentional SNARC task of Experiment 3a, except that instead of a spatial judgement a verbal Magnitude Comparison of numerical cues was required after speeded target detection (ie, is the digit cue smaller or larger than 5?). Verbal responses were recorded through a microphone for off-line scoring.

\subsubsection{Results}

Magnitude Attentional SNARC effect. 5.1\% of trials were discarded from the analyses. No error was virtually observed in the smaller/larger classification of digit cues (ie, error rate $<0.3 \%$ ). Individual average RTs were first entered in a CTI $(500,750 \mathrm{~ms}) \times$ Digit-Magnitude (small, large $) \times$ Target-Side (left, right $)$ repeated measures ANOVA. The interaction between Digit-Magnitude and Target-Side was significant $\left[F(1,23)=8.32, p<0.01, \eta^{2}=0.34\right]$ and highlighted the presence of the Att-SNARC effect. Post hoc tests showed that the Att-SNARC was significant for small digit cues though not for large ones [small digit: left target $=354 \mathrm{~ms}$ vs right $\operatorname{target}=363 \mathrm{~ms}, \quad p<0.05$; large digit: left target $=359 \mathrm{~ms}$ vs right target $=353 \mathrm{~ms}, p=0.1]$. The presence of the Att-SNARC was also qualified by a second CTI $(500,750 \mathrm{~ms}) \times$ Digit Cue $(1,2,8$, and 9) $\times$ Target-Side (left, right) ANOVA, where the Digit Cue $\times$ Target-Side interaction was significant $[F(3,69)$ $\left.=4.79, p<0.01, \eta^{2}=0.23\right]$ confirming the presence of the Att-SNARC effect (Fig. 7). The following post hoc tests showed that the Att-SNARC was significant for the 1- and 9-digit cue [1: left target $=355 \mathrm{~ms}$ vs right target $=364 \mathrm{~ms}, p<0.05$ and 9: left target $=359 \mathrm{~ms}$ vs right target $=347 \mathrm{~ms}, p<0.01]$ though not significant for the 2 [2: left target $=353 \mathrm{~ms}$ vs right target $=361 \mathrm{~ms}, p=0.07]$ and the eight-digit cues [8: left target $=360 \mathrm{~ms}$ vs right target $=359 \mathrm{~ms}, p=0.83$ ].

The regression analyses (see Fig. 8) highlighted a significant Att-SNARC effect at $500 \mathrm{~ms}$-CTI and for Collapsed-CTIs [Collapsed-CTIs: $t(23)=-2.73, p<0.01$, Slope $=-2.27, \mathrm{SD}=3.43 ; 500 \mathrm{~ms}-\mathrm{CTI}: t(23)=-3.14, p<0.01$, Slope $=-2.66$, $\mathrm{SD}=3.49$; 750 ms-CTI: $t(23)=-1.83, p=0.09$, Slope $=-1.69, \mathrm{SD}=3.82]$.

In a final analysis, we assessed the reliability of the Att-SNARC effect using the split-half method with Spearman-Brown correction. A significant correlation was found in Collapsed-CTIs condition and was not found in $500 \mathrm{~ms}-\mathrm{CTI}$ and $750 \mathrm{~ms}-$ CTI condition [Collapsed- CTIs: $r_{1,2}=0.67$, Spearman-Brown correction $=0.81$, $p<0.01$; 500 ms-CTI: $r_{1,2}=0.32$, Spearman-Brown correction $=0.48, p=0.21$; 750 ms-CTI: $r_{1,2}=0.09$, Spearman-Brown correction $\left.=0.17, p=0.72\right]$.

\subsection{EXPERIMENT 4B: EIGHT-DIGIT CUES (1, 2, 3, 4, 6, 7, 8, AND 9)}

\subsubsection{Method}

Participants. Twenty-four healthy right-handed undergraduate students (15 females, 9 males; mean age $=21.7$ years, $\mathrm{SD}=2.7$ years).

Magnitude Attentional SNARC task. The Magnitude Attentional SNARC task was the same of Experiment 4a. Stimuli, types of trials, number of trials, and training procedure were as in Experiment $1 \mathrm{~b}$. 
A

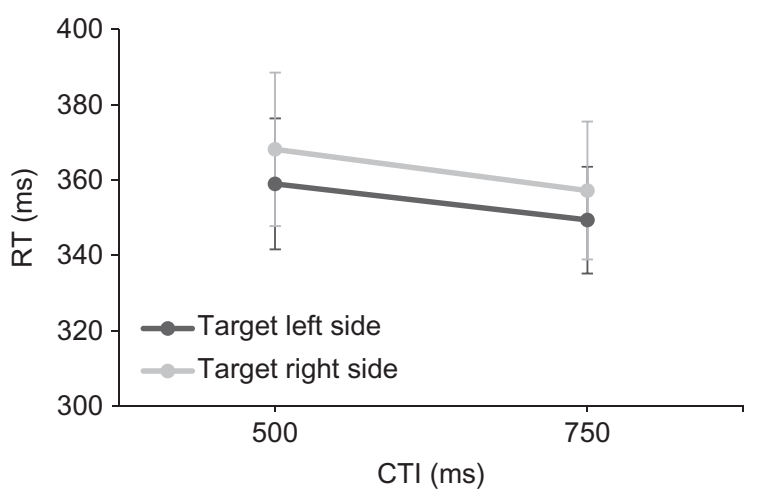

Low digit cue $(1,2)$

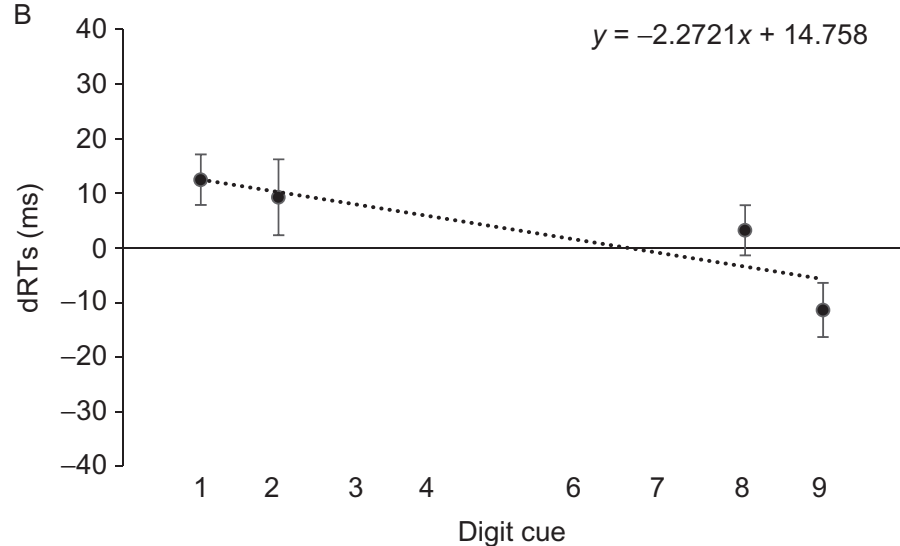

High digit cue $(8,9)$

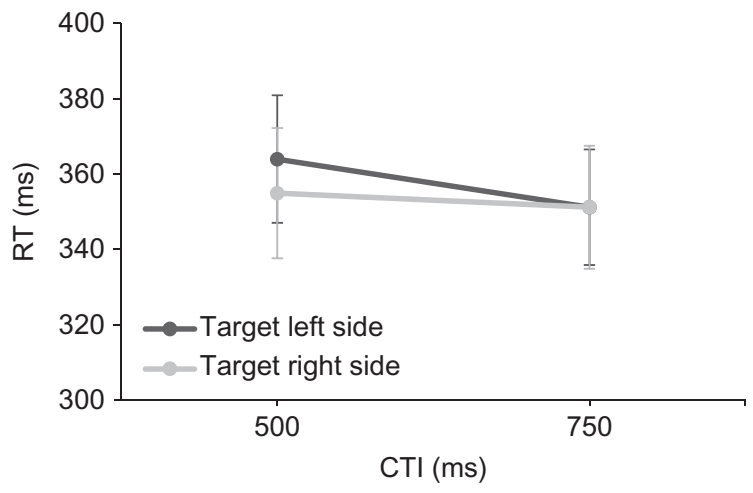

CTI (ms)

\section{FIG. 7}

Experiment 4a: Magnitude Att-SNARC task with four-digit cues. (A) Average RTs (with s.e.) to targets presented in the left and right side of space plotted as function of the magnitude of central digit cues, low (1 and 2) or high (8 and 9), and cue-target intervals. (B) Slope describing the difference between RTs to targets in the right side of space minus targets in the left side of space, plotted as function of the magnitude of central digit cues. 

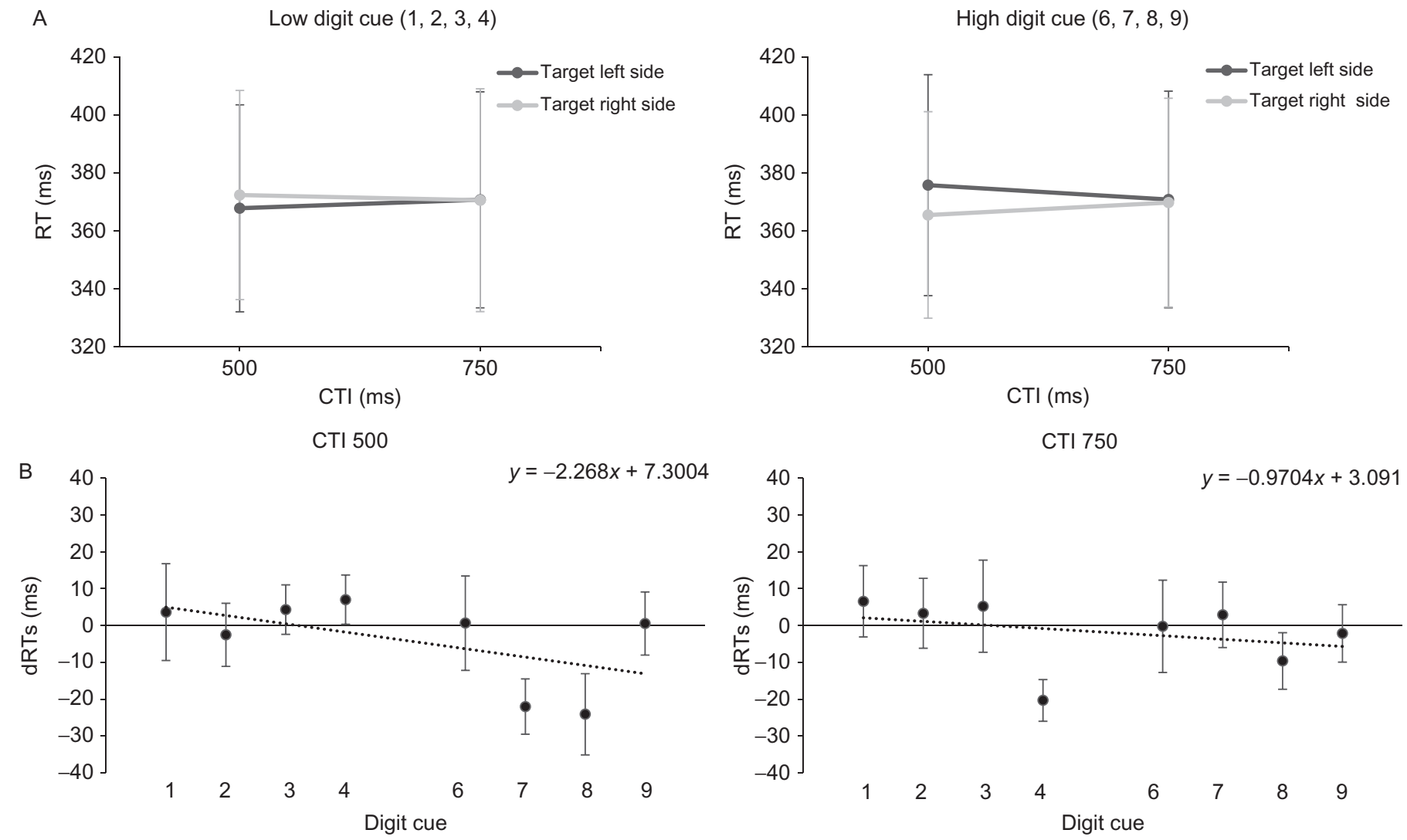

FIG. 8

Experiment 4b: Magnitude Att-SNARC task with eight-digit cues. (A) Average RTs (with s.e.) to targets presented in the left and right side of space plotted as function of the magnitude of central digit cues, low (1, 2, 3, and 4) or high (6, 7, 8, and 9), and cue-target intervals. (B) Slope describing the difference between RTs to targets in the right side of space minus targets in the left side of space, plotted as function of the magnitude of central digit cues, and cue-target intervals. 


\subsubsection{Results}

Magnitude Attentional SNARC effect. $4.9 \%$ of trials were discarded from analyses. No error was virtually observed in the small/large classification of digit cues (ie, error rate $<0.7 \%)$. Individual average RTs were first entered in a CTI $(500,750 \mathrm{~ms}) \times$ DigitMagnitude (small, large) $\times$ Target-Side (left, right) ANOVA. The interaction between Digit-Magnitude and Target-Side was not significant $[F(1,23)=1.43, p=0.25$, $\left.\eta^{2}=0.08\right]$. The interaction between CTI, Digit-Magnitude, and Target-Side resulted significant $\left[F(1,23)=7.21, p<0.01, \eta^{2}=0.30\right]$ and was qualified by a significant Att-SNARC at $500 \mathrm{~ms}-\mathrm{CTI}\left[F(1,23)=5.33, p<0.05, \eta^{2}=0.24\right]$ and no equivalent effect at $750 \mathrm{~ms}$-CTI $\left[F(1,23)=0.01, p=0.90, \eta^{2}=0\right]$. Post hoc tests showed that at $500 \mathrm{~ms}$-CTI the Att-SNARC was significant for large-magnitude numbers though not for small ones [small digit-magnitude: left target $=368 \mathrm{~ms}$ vs right target $=372 \mathrm{~ms}, \quad p=0.33$; large digit-magnitude: left target $=376 \mathrm{~ms}$ vs right target $=365 \mathrm{~ms}, p<0.05]$. The Att-SNARC was further explored in a second CTI $(500,750 \mathrm{~ms}) \times$ Digit Cue $(1,2,3,4,6,7,8$, and 9) $\times$ Target-Side (left, right) ANOVA. In this case, neither the Digit Cue $\times$ Target-Side nor the CTI $\times$ Digit Cue $\times$ Target-Side interaction resulted significant $\left[F(7,161)=1.14, p=0.34, \eta^{2}=0.06\right.$ and $F(7,161)=$ $1.77, p=0.09, \eta^{2}=0.09$, respectively]. See Fig. 8 .

The Digit Cue/Target-Side condition (Congruent, Incongruent) $\times$ Distance to reference $(1,2,3$, and 4) ANOVA highlighted a main Distance effect that did not reach significance $\left[F(3,57)=0.66, p=0.58, \eta^{2}=0.04\right]$ showing that the RTs were not modulated by the numerical distance between digit cues and central reference. The interaction between Digit Cue/Target-Side condition and Distance was not significant $\left[F(3,57)=0.57, p=0.63, \eta^{2}=0.03\right]$.

Regression analyses showed no significant Att-SNARC [Collapsed-CTIs: $t(23)=-1.41, p=0.18$, Slope $=-1.53, \mathrm{SD}=4.63$; $500 \mathrm{~ms}-\mathrm{CTI}: t(23)=-1.87$, $p=0.08, \quad$ Slope $=-2.27, \quad \mathrm{SD}=5.14 ; \quad 750 \mathrm{~ms}-\mathrm{CTI}: \quad t \quad(23)=-0.75, \quad p=0.46$, Slope $=-0.97, \mathrm{SD}=5.48]$. See Fig. 8 .

Finally, the split-half method with Spearman-Brown correction demonstrated the reliability of the Att-SNARC effect only at 500 ms-CTI $\left[r_{1,2}=0.64\right.$, SpearmanBrown correction $=0.78, p<0.01]$.

\subsubsection{Comparison of the strength of the Att-SNARC between the four- and eight-digit cues conditions}

The Magnitude Comparison Att-SNARC was not significantly different between the four- and eight-digit cues conditions $(t(38)=-0.53, p=0.6)$.

\section{COMPARING THE STRENGTH OF THE ATT-SNARC AMONG EXPERIMENTS $1-4$}

In a final series of analyses we wished to compare the strength of the Att-SNARC observed in the different experiments run in the present study. With this aim we run two separate series between-experiments ANOVA, one for the regression slopes 
observed with four-digit cues and one for those observed with eight-digit cues. With four-digit cues, the main effect of experiment was significant $[F(3,76)=16$, $\left.p<0.001, \eta^{2}=0.42\right]$. A series of $t$-tests (see Table 1) showed that the Spatial AttSNARC effect was stronger than the Magnitude Comparison Att-SNARC and that both effects were stronger than the Att-SNARC and the Imagery Att-SNARC. No difference was found between the Att-SNARC and the Imagery Att-SNARC. Also with eight-digit cues, the main effect of Experiment was significant $[F(3,76)=4.5$, $p<0.01, \eta^{2}=0.16$ ]. $t$-Tests (see Table 2) showed that the strength of the effect was

Table $1 t$-Test Among the Regression Slopes (Experiments 1a, 2a, 3a, and 4a) Describing the Attentional SNARC, the Imagery Att-SNARC, the Spatial AttSNARC, and the Magnitude Att-SNARC Effects

\begin{tabular}{l|l|l|l}
$\begin{array}{l}\text { Four-Digit Cues } \\
(\mathbf{1}, \mathbf{2}, \mathbf{8} \text {, and } \mathbf{9 )}\end{array}$ & $\begin{array}{l}\text { 1. Attentional } \\
\text { SNARC }\end{array}$ & $\begin{array}{l}\text { 2. Imagery } \\
\text { Att-SNARC }\end{array}$ & $\begin{array}{l}\text { 3. Spatial } \\
\text { Att-SNARC }\end{array}$ \\
$\begin{array}{l}\text { 1. Attentional SNARC } \\
\text { Slope }=1.01 \text { (n.s.) }\end{array}$ & - & \\
$\begin{array}{l}\text { 2. Imagery Att-SNARC } \\
\text { Slope }=0.54 \text { (n.s.) }\end{array}$ & $p=0.45$ & - & \\
$\begin{array}{l}\text { 3. Spatial Att-SNARC } \\
\text { Slope }=-\mathbf{4 . 8}(\boldsymbol{p}<\mathbf{0 . 0 0 1 )}\end{array}$ & $\boldsymbol{p}<\mathbf{0 . 0 0 1}$ & $\boldsymbol{p}<\mathbf{0 . 0 0 1}$ & - \\
$\begin{array}{l}\text { 4. Magnitude Att-SNARC } \\
\text { Slope }=-\mathbf{2 . 2 7}(\boldsymbol{p}<\mathbf{0 . 0 1})\end{array}$ & $\boldsymbol{p}<\mathbf{0 . 0 1}$ & $\boldsymbol{p}<\mathbf{0 . 0 1}$ & $\boldsymbol{p}<\mathbf{0 . 0 5}$ \\
\hline
\end{tabular}

Significant differences are reported in bold format.

Table $2 t$-Test Among the Regression Slopes (Experiment 1b, 2b, 3b, and 4b) Describing the Attentional SNARC, the Imagery Att-SNARC, the Spatial AttSNARC, and the Magnitude Att-SNARC Effects

\begin{tabular}{l|l|l|l}
$\begin{array}{l}\text { Eight-Digit Cues } \\
(\mathbf{1}, \mathbf{2}, \mathbf{3}, \mathbf{4}, \mathbf{6}, \mathbf{7}, \mathbf{8} \text {, and 9) }\end{array}$ & $\begin{array}{l}\text { 1. Attentional } \\
\text { SNARC }\end{array}$ & $\begin{array}{l}\text { 2. Imagery } \\
\text { Att-SNARC }\end{array}$ & $\begin{array}{l}\text { 3. Spatial } \\
\text { Att-SNARC }\end{array}$ \\
$\begin{array}{l}\text { 1. Attentional SNARC } \\
\text { Slope }=\mathbf{0 . 9 7}(\boldsymbol{p}<\mathbf{0 . 0 5})\end{array}$ & - & & \\
$\begin{array}{l}\text { 2. Imagery Att-SNARC } \\
\quad \text { Slope }=-1 \text { (n.s.) }\end{array}$ & $\boldsymbol{p}<\mathbf{0 . 0 5}$ & - & \\
$\begin{array}{l}\text { 3. Spatial Att-SNARC } \\
\text { Slope }=-\mathbf{2 . 9}(\boldsymbol{p}<\mathbf{0 . 0 1})\end{array}$ & $\boldsymbol{p}<\mathbf{0 . 0 0 1}$ & $p=0.15$ & - \\
$\begin{array}{l}\text { 4. Magnitude Att-SNARC } \\
\text { Slope }=-1.53 \text { (n.s.) }\end{array}$ & $\boldsymbol{p}<\mathbf{0 . 0 5}$ & $p=0.71$ & $p=0.33$ \\
\hline
\end{tabular}

Significant differences are reported in bold format. 
significantly different between the Att-SNARC and the other tasks while no significant difference was found among the strength of the Imagery, Spatial, and Magnitude Comparison Att-SNARC.

\section{DISCUSSION}

The results of these four experiments allow drawing a number of important conclusions on the functional bases of number-space interaction in humans. First, in agreement with other recent investigations, in Experiments 1a and 1b we have reconfirmed that the mere perception of Arabic digits does not trigger concomitant shifts of spatial attention. This negative finding points at a basic functional dissociation between number and space coding. Second, in Experiment $3 \mathrm{a}$ and $3 \mathrm{~b}$ a clear interaction between number processing and lateral leftward/rightward shift of attention was found when the task required the spatial coding of numbers with respect to a central numerical reference. On the one hand, in line with arguments that we have advanced in previous papers (Aiello et al., 2012; Fattorini et al., 2015b; Rotondaro et al., 2015), the results of Experiment 1 are in keeping with the dissociation between pathological spatial and numerical biases that is observed in right brain damaged patients with left spatial neglect (for review, see Aiello et al., 2012; Fattorini et al., 2015b) when they are engaged in the mental bisection of number intervals (Zorzi et al., 2002), which is a task that does not require the association of spatial codes to number magnitudes. The same dissociation is also in agreement with recent 7T-fMRI findings showing no relationship between numerosity or Arabic coding and direction of visuospatial responses of topographically organized number-sensitive neurons in the human parietal cortex (Harvey et al., 2013). On the other hand, the clear numberspace interaction highlighted in Experiment 3, agrees with homogenous findings from different authors (for review, see Aiello et al., 2012; Fattorini et al., 2015b) showing that during the performance of SNARC tasks, when the association of left/right spatial codes to number magnitude is explicitly required for the selection of motor responses, patients with left spatial neglect show faster reaction time to numbers immediately higher, ie, to the right in this case, of the central reference.

The results of Experiments 2 and 3 offer relevant clues on the type of cognitive processing that is required to make digit cues able of triggering shifts of spatial attention. In Experiment 2, participants were asked to imagine cues on horizontal $10 \mathrm{~cm}$ ruler: in this case no evidence of the Att-SNARC was found. One weakness of Experiment 2 is that we had no independent evidence that participants were actually using visual-spatial imagery in the performance of the task. The only clue that suggests this possibility is that in the task with eight-digit cues we found that RTs to lateral targets were almost modulated as a continuous function of the numerical distance between the digit cue and the central numerical reference on the MNL: this distance effect, however, only showed a tendency toward significance (ie, $p=0.1$ ). In contrast to this, in Experiment 3 the Att-SNARC was evident when an imaginative 
numerical reference, ie, the number " 5 ," was provided and participants were required to explicitly associate "left" vs "right" spatial codes with number magnitudes to decide whether a digit cue is positioned to the left or to the right of number " 5 ." This result provides support to our previous conclusion that number-space interactions are mainly driven by the use of spatial codes in the task at hand. Interestingly, like in our previous study (Fattorini et al., 2015b), split-half testing showed that the significant Att-SNARC was not fully reliable, which is in contrast to the high reliability of the conventional PJand MC-SNARC effects (see Fattorini et al., 2015b). All together these result from the Spatial Att-SNARC and the SNARC tasks suggest that number-space interactions can be elicited both when left/right spatial codes are directly associated to numbers, as in the Spatial Att-SNARC, and when they are associated indirectly to numbers via selection of the motor response, as in conventional PJ- and MC-SNARC tasks. Nonetheless the aforementioned high reliability of the SNARC effect, as compared to poorer reliability of the Spatial Att-SNARC (Fattorini et al., 2015b) suggests that the indirect association elicits a more stable interaction between representations of numbers and representation of space.

Compared with the results of Experiment 3, Experiments 4a and 4b provided mixed findings. The postdetection Magnitude Comparison of digit cues with the reference "5" produced a significant Att-SNARC with a set of four-digit cues (Experiment 4a) although when a larger set of eight cues was used (Experiment 4b), the Att-SNARC was only evident at $500 \mathrm{~ms}-\mathrm{CTI}$ and only with large digit cues. These results are at variance with those of the Experiment 3 in which a significant general Spatial Att-SNARC effect was found independently of the set of cues and CTIs. The nonhomogenous results of our Experiment 4 are generally in line with those of the study by Zanolie and Pecher (2014) who, using a set of four-digit cues, found a significant Magnitude Comparison Att-SNARC in their Experiment 3 without replicating the same result in their Experiment 6 . In addition, it is worth mentioning that in a new combined behavioral-ERPs study in preparation (Fattorini et al., 2015a), we were unable to replicate the Magnitude Comparison Att-SNARC, while we replicated once more the Spatial Att-SNARC.

Our findings highlighted two important differences between the Spatial and the Magnitude Comparison Att-SNARC. First, with four-digit cues the Spatial AttSNARC was stronger than the Magnitude Comparison one. Second, only in the case of the Att-SNARC RTs to lateral targets were significantly modulated by the numerical distance between the digit cue and the central reference, so that the larger was the distance the faster were RTs. This effect was present both for congruent trials, in which targets were presented on the same side of space occupied by the central digit cue on the MNL, and for incongruent trials in which targets were presented on the side of space opposite to that occupied by the central digit cue on the MNL. This latter finding suggests that the cognitive load of the spatial decision, with decisions being easier the larger the distance between the digit cue and the reference, adds to the speed of target detection independently of their spatial congruency with the lateral position of the digit cue on the MNL. 
Can this set of findings on the Att-SNARC provide hints on the functional origin of the SNARC effect? In a previous study we have found that interindividual variations in the strength of the Att-SNARC bear no relationship with the strength of the SNARC measured in the same individuals. In light of the findings of the present study, it could be interesting to assess the relationships between the strength of the significant Spatial Att-SNARC effect and that of the PJ- or MC-SNARC effects. This would clarify whether the strength of the direct link established during the performance of the Spatial Att-SNARC between left/right spatial codes and number magnitudes is correlated with the strength of the indirect link established, through the selection of motor responses, between left/right spatial codes and number magnitudes during the performance of SNARC tasks.

\section{CONCLUSIONS}

Several pieces of evidence that we have reviewed in the introduction of the present chapter, point at the nonautomaticity and instability of the functional link between number and space processing. The empirical findings of our four experiments corroborate this view and show that the widespread conviction, prompted by the reliability of the SNARC effect, that the representation of numbers in the human brain has an inherent directional-spatial component should be profoundly revised. This influential belief has generated disparate empirical observations that in some cases have not resisted to independent replications or have not been the object of adequate replications. This is to say that one should not refrain from exploring and appreciating the complexity of the brain mechanisms that by setting an adaptive and "ad hoc" interplay between numerical and spatial thinking facilitate the performance of everyday simple computational activities and the achievement of important progresses in mathematics.

\section{REFERENCES}

Aiello, M., Jacquin-Courtois, S., Merola, S., Ottaviani, T., Tomaiuolo, F., Bueti, D., et al., 2012. No inherent left and right side in human 'mental number line': evidence from right brain damage. Brain 135, 2492-2505. http://dx.doi.org/10.1093/brain/aws114. (first published online May 10, 2012).

Bertillon, J., 1880. De la vision des nombres. La Nature 378, 196-198.

Bertillon, J., 1881. De la vision et la memoire des nombres. La Nature 408, 202-203.

Bertillon, J., 1882. De la vision des nombres. La Nature, 428, 265-268.

Bonato, M., Priftis, K., Marenzi, R., Zorzi, M., 2009. Normal and impaired reflexive orienting of attention after central nonpredictive cues. J. Cogn. Neurosci. 21 (4), 745-759. http://dx.doi.org/10.1162/jocn.2009.21. 
Casarotti, M., Michielin, M., Zorzi, M., Umiltà, C., 2007. Temporal order judgment reveals how number magnitude affects visuospatial attention. Cognition 1, 101-117. http://dx.doi.org/ 10.1016/j.cognition.2006.09.001.

Cohen Kadosh, R., Lammertyn, J., Izard, V., 2008. Are numbers special? An overview of chronometric, neuroimaging, developmental and comparative studies of magnitude representation. Prog. Neurobiol. 84, 132-147. http://dx.doi.org/10.1016/j.pneurobio. 2007.11.001.

Dehaene, S., Dupoux, E., Mehler, J., 1990. Is numerical comparison digital? Analogical and symbolic effects in two-digit number comparison. J. Exp. Psychol. Hum. Percept. Perform. 16, 626-641. http://dx.doi.org/10.1037/0096-1523.16.3.626.

Dehaene, S., Bossini, S., Giraux, P., 1993. The mental representation of parity and number magnitude. J. Exp. Psychol. Gen. 122, 371-396. http://dx.doi.org/10.1037/0096-3445.122.3.371.

Dodd, M.D., Van der Stigchel, S., Leghari, M.A., Fung, G., Kingstone, A., 2008. Attentional SNARC: there's something special about numbers (let us count the ways). Cognition 108, 810-818. http://dx.doi.org/10.1016/j.cognition.2008.04.006.

Eagleman, D.M., 2009. The objectification of overlearned sequences: a new view of spatial sequence synesthesia. Cortex 45 (10), 1266-1277. http://dx.doi.org/10.1016/j. cortex.2009.06.012.

Fattorini, E., Pinto, M., D’Onofrio, M., Doricchi, F., 2015a. Number-Space Interaction Is Not Automatic: An ERP Study on the Attentional SNARC. Abstract submitted to the EWCN 2016, Bressanone, Italy.

Fattorini, E., Pinto, M., Rotondaro, F., Doricchi, F., 2015b. Perceiving numbers does not cause automatic shifts of spatial attention. Cortex 73, 298-316. http://dx.doi.org/10.1016/j. cortex.2015.09.007.

Fischer, M.H., Castel, A.D., Dodd, M.D., Pratt, J., 2003. Perceiving numbers causes spatial shifts of attention. Nat. Neurosci. 6, 555-556. http://dx.doi.org/10.1038/nn1066.

Galfano, G., Rusconi, E., Umilta‘, C., 2006. Number magnitude orients attention, but not against one's will. Psychon. Bull. Rev. 13, 869-874. http://dx.doi.org/10.3758/BF03194011.

Galton, F., 1880a. Visualised numerals. Nature 21, 252-256.

Galton, F., 1880b. Visualised numerals. Nature 21, 494-495.

Gevers, W., Ratinckx, E., De Baene, W., Fias, W., 2006. Further evidence that the SNARC effect is processed along a dual-route architecture: evidence from the lateralized readiness potential. Exp. Psychol. 53 (1), 58-68.

Gevers, W., Santens, S., Dhooge, E., Chen, Q., Van den Bossche, L., Fias, W., 2010. Verbal-spatial and visuo-spatial coding of number-space interactions. J. Exp. Psychol. Gen. 139, 180-190. http://dx.doi.org/10.1037/a0017688.

Goffaux, V., Martin, R., Dormal, G., Goebel, R., Schiltz, C., 2012. Attentional shifts induced by uninformative number symbols modulate neural activity in human occipital cortex. Neuropsychologia 50 (14), 3419-3428. http://dx.doi.org/10.1016/j.neuropsychologia.2012.09.046.

Harvey, B.M., Klein, B.P., Petridou, N., Dumoulin, S.O., 2013. Topographic representation of numerosity in the human parietal cortex. Science 341 (6150), 1123-1126. http://dx.doi. org/10.1126/science.1239052.

Herrera, A., Macizo, P., Semenza, C., 2008. The role of working memory in the association between number magnitude and space. Acta Psychol. 128 (2), 225-237. http://dx.doi.org/ 10.1016/j.actpsy.2008.01.002.

Hubbard, E.M., Piazza, M., Pinel, P., Dehaene, S., 2005. Interactions between number and space in parietal cortex. Nat. Rev. Neurosci. 6 (6), 435-448. http://dx.doi.org/10.1038/ nrn1684. 
Hubbard, E.M., Ranzini, M., Piazza, M., Dehaene, S., 2009. What information is critical to elicit interference in number-form synaesthesia? Cortex 45 (10), 1200-1216.

Ishihara, M., Jacquin-Courtois, S., Flory, V., Salemme, R., Imanaka, K., Rossetti, Y., 2006. Interaction between space and number representations during motor preparation in manual aiming. Neuropsychologia 44 (7), 1009-1016. http://dx.doi.org/10.1016/j. neuropsychologia.2005.11.008.

Jarick, M., Dixon, M.J., Maxwell, E.C., Nicholls, M.E., Smilek, D., 2009. The ups and downs (and lefts and rights) of synaesthetic number forms: validation from spatial cueing and SNARC-type tasks. Cortex 45 (10), 1190-1199. http://dx.doi.org/10.1016/j. cortex.2009.04.015.

Keus, I.M., Schwarz, W., 2005. Searching for the functional locus of the SNARC effect: evidence for a response related origin. Mem. Cognit. 33, 681-695. http://dx.doi.org/ 10.3758/BF03195335.

Lorch Jr., R.F., Myers, J.L., 1990. Regression analyses of repeated measures data in cognition research. J. Exp. Psychol. Learn. Mem. Cogn. 16, 149-157. http://dx.doi.org/10.1037/02787393.16.1.149.

Moyer, R.S., Landauer, T.K., 1967. Time required for judgements of numerical inequality. Nature 215, 1519-1520. http://dx.doi.org/10.1038/2151519a0.

Müller, D., Schwarz, W., 2007. Exploring the mental number line: evidence from a dual-task paradigm. Psychol. Res. 71 (5), 598-613. http://dx.doi.org/10.1007/s00426-006-0070-6.

Price, M.C., Mattingley, J.B., 2013. Automaticity in sequence-space synaesthesia: a critical appraisal of the evidence. Cortex 49 (5), 1165-1186.

Proctor, R.W., Cho, Y.S., 2006. Polarity correspondence: a general principle for performance of speeded binary classification tasks. Psychol. Bull. 132, 416-442. http://dx.doi.org/ 10.1037/0033-2909.132.3.416.

Ranzini, M., Dehaene, S., Piazza, M., Hubbard, E.M., 2009. Neural mechanisms of attentional shifts due to irrelevant spatial and numerical cues. Neuropsychologia 47, 2615-2624. http://dx.doi.org/10.1016/j.neuropsychologia.2009.05.011.

Ristic, J., Wright, A., Kingstone, A., 2006. The number line reflects top-down control. Psychon. Bull. Rev. 13, 862-868. http://dx.doi.org/10.3758/BF03194010.

Rossetti, Y., Jacquin-Courtois, S., Aiello, M., Ishihara, M., Brozzoli, C., Doricchi, F., 2011. Neglect "around the clock": dissociating number and spatial neglect in right brain damage. In: Dehaene, S., Brannon, E. (Eds.), Space, Time and Number in the Brain e Searching for Evolutionary Foundations of Mathematical Thought: Attention and Performance XXIV. Elsevier Science, Amsterdam, pp. 149-172.

Rotondaro, F., Merola, S., Aiello, M., Pinto, M., Doricchi, F., 2015. Dissociation between line bisection and mental-number-line bisection in healthy adults. Neuropsychologia $75,565-576$.

Sagiv, N., Simner, J., Collins, J., Butterworth, B., Ward, J., 2006. What is the relationship between synaesthesia and visuo-spatial number forms? Cognition 101 (1), 114-128. http://dx.doi.org/10.1016/j. cognition .2005.09.004.

Salillas, E., El Yagoubi, R., Semenza, C., 2008. Sensory and cognitive processes of shifts of spatial attention induced by numbers: an ERP study. Cortex 44 (4), 406-413. http://dx.doi. org/10.1016/j.cortex.2007.08.006.

Santens, S., Gevers, W., 2008. The SNARC effect does not imply a mental number line. Cognition 108 (1), 263-270. http://dx.doi.org/10.1016/j.cognition.2008.01.002.

Schneider, W., Eschman, A., Zuccolotto, A., 2002. E-Prime User's Guide. Psychology Software Tools, Pittsburgh, PA. 


\section{CHAPTER 9 Instability of number-space interaction}

Schuller, A.M., Hoffmann, D., Goffaux, V., Schiltz, C., 2015. Shifts of spatial attention cued by irrelevant numbers: electrophysiological evidence from a target discrimination task. J. Cogn. Psychol. 27 (4), 442-458.

Seron, X., Pesenti, M., Noël, M.P., Deloche, G., Cornet, J.A., 1992. Images of numbers, or "when 98 is upper left and 6 sky blue" Cognition 44 (1), 159-196. http://dx.doi.org/ 10.1016/0010-0277(92)90053-K.

van Dijck, J.P., Fias, W., 2011. A working memory account for spatial-numerical associations. Cognition 119 (1), 114-119. http://dx.doi.org/10.1016/j.cognition.2010.12.013.

van Dijck, J.P., Gevers, W., Fias, W., 2009. Numbers are associated with different types of spatial information depending on the task. Cognition 113 (2), 248-253. http://dx.doi. org/10.1016/j.neuropsychologia.2011.05.005.

van Dijck, J.P., Abrahamse, E., Majerus, S., Fias, W., 2013. Spatial attention interacts with serial order retrieval from verbal working memory. Psychol. Sci. 24, 1854-1859. http:// dx.doi.org/10.1177/0956797613479610.

van Dijck, J.P., Abrahamse, E.L., Acar, F., Ketels, B., Fias, W., 2014. A working memory account of the interaction between numbers and spatial attention. Q. J. Exp. Psychol. 67 (8), 1500-1513. http://dx.doi.org/10.1080/17470218.2014.903984.

Wood, G., Nuerk, H.-C., Willmes, K., Fischer, M.H., 2008. On the cognitive link between space and number: a meta-analysis of the SNARC effect. Psychol. Sci. Quart. 50 (4), 489-525.

Zanolie, K., Pecher, D., 2014. Number-induced shifts in spatial attention: a replication study. Front. Psychol. 5 (987), 10-3389.

Zorzi, M., Priftis, K., Umilta, C., 2002. Brain damage: neglect disrupts the mental number line. Nature 417 (6885), 138-139. http://dx.doi.org/10.1038/417138. 